hep-th/0304192

PUPT-2082

\title{
Closed Strings as Imaginary D-branes
}

\author{
Davide Gaiotto, Nissan Itzhaki and Leonardo Rastelli \\ Physics Department, Princeton University, Princeton, NJ 08544
}

\begin{abstract}
Sen has recently drawn attention to an exact time-dependent Boundary Conformal Field Theory with the space-time interpretation of brane creation and annihilation. An interesting limit of this BCFT is formally equivalent to an array of D-branes located in imaginary time. This raises the question: what is the meaning of D-branes in imaginary time? The answer we propose is that D-branes in imaginary time define purely closed string backgrounds. In particular we prove that the disk scattering amplitude of $m$ closed strings off an arbitrary configuration of imaginary branes is equivalent to a sphere amplitude with $m+1$ closed string insertions. The extra puncture is a specific closed string state, generically normalizable, that depends on the details of the brane configuration. We study in some detail the special case of the array of imaginary D-branes related to Sen's BCFT and comment on its space-time interpretation. We point out that a certain limit of our set-up allows to study classical black hole creation and suggests a relation between Choptuik's critical behavior and a phase-transition à la Gregory-Laflamme. We speculate that open string field theory on imaginary D-branes is dual to string theory on the corresponding closed string background.
\end{abstract}




\section{Contents}

\begin{tabular}{lll}
\hline & Introduction & 3
\end{tabular}

2 Preliminaries $\quad 6$

2.1 Naive argument . . . . . . . . . . . . . . . . . . . . 6

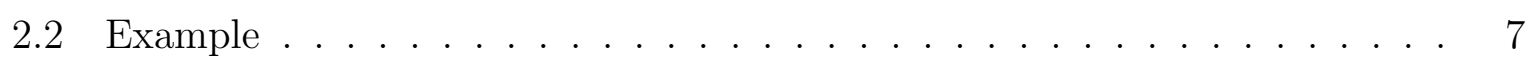

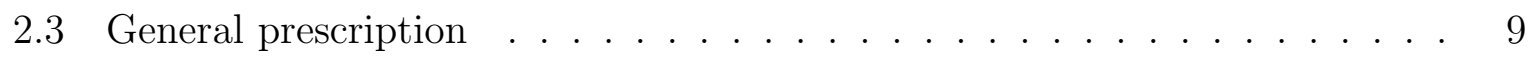

3 Disk amplitudes $\quad 10$

3.1 Tachvon two-point amplitude . . . . . . . . . . . . . . . . 10

3.2 Boundary state computation . . . . . . . . . . . . . . . 13

3.3 Higher-point disk amplitudes . . . . . . . . . . . . . . . . . . . . . . 15

3.4 Interpretation . . . . . . . . . . . . . . . . . . . . . . . 16

4 The closed string state $\quad 18$

4.1 Massless secton . . . . . . . . . . . . . . . . . . . . 18

4.2 Massive modes . . . . . . . . . . . . . . . . . . . . . . . 20

5 On open and closed string moduli

$6 \quad$ A more general set-up $\quad 26$

6.1 Superstring . . . . . . . . . . . . . . . . . . . . 28

7 Open string field theory $\quad 28$

$8 \mathrm{a}=2 \pi$ and reconstruction of the brane 30

8.1 Smearing and brane creation . . . . . . . . . . . . . . . 30

9 From Choptuik to Gregorv-Laflamme $\quad 33$ 


\section{Introduction}

Worldsheet duality between open and closed strings is one of the truly fundamental ideas of string theory. Many modern developments have originated from the application of this idea to supersymmetric configurations of D-branes. In this paper we show that the study of non-supersymmetric, unstable D-branes gives some clues for a new intriguing incarnation of open/closed string duality.

Our starting point is the real-time process of D-brane creation and annihilation, which has recently attracted much attention [1]-21]. In particular, Sen 2] introduced a simple class of models in bosonic string theory obtained by perturbing the flat-space $c=26 \mathrm{CFT}$ with the exactly marginal deformation

$$
\lambda \int d t \cosh \left(X^{0}(t)\right)
$$

where $t$ is a coordinate on the worldsheet boundary, and $\lambda$ is a free parameter in the range $0 \leq \lambda \leq \frac{1}{2}$. This is a family of exact solutions of classical open string theory whose space-time picture is that of an unstable brane being created at a time $X^{0} \sim-\tau$ and decaying at a time $X^{0} \sim+\tau$, with $\tau=-\log (\sin (\pi \lambda))$. For $\lambda=\frac{1}{2}$ the lifetime of the brane is zero, that is, there is no brane to be found anywhere. Moreover, the corresponding boundary state appears to vanish identically [2]. This is fascinating as it seems to suggest that for $\lambda=\frac{1}{2}$ the BCFT (1.1) describes the stable closed string vacuum, where open string degrees of freedom are absent. Somehow the boundary perturbation (1.1) with $\lambda=\frac{1}{2}$ must get rid of the worldsheet boundary!

In the framework of (1.1), we have the opportunity to precisely test a scenario [22, 23] for how purely closed string amplitudes may be obtained at the tachyon vacuum. The basic idea is that as the tachyon condenses, worldsheets with large holes are suppressed, and the integration over moduli space should localize to the region where the holes shrink to points. This heuristic picture was made somewhat more concrete in [23], where it was argued that amplitudes for $m$ external closed strings on the disk reduce at the tachyon vacuum to sphere amplitudes with the same $m$ closed string punctures plus an additional insertion of a zero-momentum state (possibly a soft dilaton), a remnant of the shrunk boundary. This analysis [23] was performed in the framework of a regulated version of vacuum string field theory [24]. However, due to subtleties in the regulation procedure, it was difficult to make this conclusion completely precise. 
Since we wish to focus on the case $\lambda=\frac{1}{2}$, it is very useful to realize that at this critical value the BCFT admits a simple description. By Wick rotation $X^{0} \rightarrow i X$, one obtains the well-known exactly marginal deformation $\lambda \cos (X)$ [25] (infact this is how Sen arrived at (1.1) in the first place), which for $\lambda=\frac{1}{2}$ is equivalent to an infinite array of D-branes located at $X=2 \pi\left(n+\frac{1}{2}\right)$ [25, 26]. We could thus say that at the critical value $\lambda=\frac{1}{2}$, the time-dependent boundary deformation (1.1) becomes an array of D-branes located at imaginary times

$$
X^{0}=i 2 \pi\left(n+\frac{1}{2}\right), \quad n \in \mathbf{Z} .
$$

This is an empty statement if we do not define the meaning of D-branes in imaginary time. Our approach to that issue is very simple: Any quantity that one wishes to compute for a configuration of D-branes in imaginary time should be obtained by Wick rotation of the configuration in real space. This prescription gives consistent answers with an interesting physical meaning.

A natural class of observables is given by scattering amplitudes of closed strings on the disk in the background of the brane configuration (1.2). We find that these reduce to sphere amplitudes with $m+1$ punctures. The extra puncture is, however, not a soft dilaton as in [23], but a non-trivial closed string state that involves the whole tower of massive modes. Thus the BCFT (1.1) with $\lambda=\frac{1}{2}$ describes a purely closed string background with no physical open string degrees of freedom. This background, however, is not the closed string vacuum, but a specific time-dependent state with non-zero energy. The detailed features of this background are very reminiscent of 'tachyon matter' [3, 4].

While these results were first obtained in the special case (1.2), the basic conclusion is much more general. A generic configuration of imaginary D-branes defines a closed string background. The details of the background depend on the details of the configuration of imaginary D-branes. Exactly marginal open string deformations, for example deformations that move the positions of the imaginary branes, are naturally reinterpreted as deformations of the closed string background. The case (1.2) is seen to be very special as the closed string state has divergent norm [12, and the background admits an additional exactly marginal deformation which is associated with the creation of an actual brane in real time.

An outline of the paper is as follows. In section 2 we spell out the basic prescription for how to deal with the array of D-branes in imaginary time. We consider a more general 
case in which $X^{0}=i a\left(n+\frac{1}{2}\right)$, where $a$ is an arbitrary parameter, and find a general formula for disk amplitudes associated with such an array. In section 3 we analyze in detail disk amplitudes for scattering of $m$ external closed strings from the array of Dbranes in imaginary time. By an exact computation, we show that they are equivalent to sphere amplitudes with the same $m$ closed strings insertions plus the insertion of an extra closed string state $|W\rangle$. The details of this closed string state, in particular its space-time interpretation, are studied in section 4. The energy of the state is finite and of order $O\left(g_{s}^{0}\right)=O(1)$ for any $a>2 \pi$. The case $a=2 \pi$, corresponding to $\lambda=\frac{1}{2}$ in the BCFT (1.1), is seen to be special as the normalization and energy of the state diverge [12. We suggest a heuristic mechanism to cutoff this divergence, namely we point out that the gravitational back-reaction makes the effective distance $a_{\text {eff }}=2 \pi+\gamma g_{s}$, with $\gamma>0$, leading to a total energy of order $1 / g_{s}$.

In section 5 it is shown that although the imaginary array of branes does not have propagating open string degrees of freedom, open strings still play an important role. There still exists a discrete set of on-shell open string vertex operators corresponding to exactly marginal deformations, for example deformations that move the branes around in imaginary time. These open string moduli are re-interpreted as closed string deformations, according to a precise dictionary. In section 6 we show that, subject to certain reality conditions, one can distribute D-branes quite freely in the complex $X^{0}$ plane. The reduction of disk amplitudes to sphere amplitudes still holds in this general case. We briefly comment on extensions to the superstring. In section 7 we briefly discuss some ideas about the open string field theory associated with D-branes in imaginary time and speculate that a version of vacuum string field theory may be obtained in the limit $a \rightarrow \infty$.

Section 8 is devoted to the case $a=2 \pi$. In this case there is an additional exactly marginal open string deformation (which we may label as ' $\left.\cosh \left(X^{0}\right)^{\prime}\right)$ which is not dual to a purely closed deformation, as it introduces instead an actual D-brane in real time. We give a treatment of (1.1) for all $\lambda \leq \frac{1}{2}$ by representing the boundary state as an infinite array of some specific smeared sources, and then performing the Wick rotation. We find that for $\lambda<\frac{1}{2}$ a time-delay of order $\tau$ is introduced between the incoming and the outgoing parts of the closed string wave, while for $\left|X^{0}\right|<\tau$ there is an actual source for the closed string fields.

Finally in section 9 we describe a curious application of our set-up. We consider an array of branes in imaginary time, and tune various parameters in such a way that 
the closed string state $|W\rangle$ becomes simply a classical, spherically symmetric dilaton wave with barely enough energy to form a black hole. Fascinating critical behavior was discovered by Choptuik [27] in such a system. We observe that in the corresponding Euclidean theory this critical point corresponds to a phase transition reminiscent of the Gregory-Laflamme [28] phase transition. We speculate on a possible realization of this phase transition in large $N$ open string field theory.

While this paper was in preparation we received [12] that has some overlapping results.

\section{Preliminaries}

We wish to give a meaning to the notion of an array of D-branes located at imaginary times $X^{0}=i\left(n+\frac{1}{2}\right) a$. The distance $a=2 \pi$ (in units $\alpha^{\prime}=1$ ) corresponds to the $\lambda=\frac{1}{2}$ case of the BCFT (1.1), but it is interesting and not more difficult to keep $a$ arbitrary. In this section we define our basic prescription to compute disk amplitudes of external closed strings in the background of this 'imaginary array'. We first give a naive argument why all such scattering amplitudes vanish. Then we illustrate, via a simple example, a natural analytic continuation prescription that actually yields non-zero answers. Finally we derive a simple general formula that expresses the scattering amplitude $S$ in the background of the imaginary array in terms of the scattering amplitude $\widetilde{A}$ for a single D-brane in real time.

\subsection{Naive argument}

Let us denote the scattering amplitude of some closed strings off a single D-brane located at $X=0$ by $\widetilde{A}$. Here $X$ is a spatial coordinate. To find the scattering amplitude $S$ for an array of D-branes located at imaginary time we could proceed in two steps:

(i) Find the scattering amplitude $\widetilde{S}$ for an array of D-branes located at the real positions $X=\left(n+\frac{1}{2}\right) a, n \in \mathbf{Z}$. This is given by

$$
\widetilde{S}(P, \ldots)=\sum_{n=-\infty}^{\infty} \widetilde{A}(P, \ldots) e^{i\left(n+\frac{1}{2}\right) a P}=\widetilde{A}(P, \ldots) \sum_{n=-\infty}^{\infty}(-1)^{n} 2 \pi \delta(P a-2 \pi n),
$$

where $P$ is the total momentum in the $X$ direction and the dots denote other variables that the amplitude may depend on. This is a precise equality in the sense of distributions. Simply put, the momentum has to be quantized due to the periodicity in $X$. 
(ii) Apply (inverse) Wick rotation

$$
X \rightarrow-i X^{0}, \quad P \rightarrow i E
$$

where now $X^{0}$ and $E$ are real. This has the effect of turning the spatial coordinate $X$ into a temporal coordinate $X^{0}$ and of rotating the array of $\mathrm{D}$-branes from the real axis to the imaginary axis, $X^{0}=i\left(n+\frac{1}{2}\right) a$. So it takes us exactly to the set-up that we wish to study. Wick rotating (2.1) we get the formal expression

$$
S(E, \ldots) \equiv \widetilde{S}(i E, \ldots)=\widetilde{A}(i E, \ldots) \sum_{n=-\infty}^{\infty}(-1)^{n} 2 \pi \delta(i E a-n) .
$$

Naively this implies that $S(E, \ldots)$ is identically zero since for any real $E$ the delta func-

tions vanish. However one has to be more careful, as $\widetilde{A}(i E, \ldots)$ may blow up for some real values of $E$ yielding a non-zero $S(E)$. Clearly the discussion so far has been quite formal, for example the summation (2.1) does not commute with the Wick rotation (2.2) in the sense that if we first Wick rotate and then sum over the array, the sum does not converge for any real $E$. We need to specify an unambiguous prescription for the analytic continuation (2.2). Let us illustrate how a natural prescription comes about in a simple example.

\subsection{Example}

The example we wish to study is

$$
\widetilde{A}(P, \ldots)=\frac{1}{P^{2}+c^{2}},
$$

where $c$ is a real number (we take for definiteness $c>0$ ) that can depend on the other variables but not on $P$. This is the one-dimensional Euclidean propagator. In position space,

$$
\widetilde{G}(X)=\int d P \widetilde{A}(P) e^{i P X}=\frac{\pi}{c} e^{-c|X|},
$$

which obeys $\left(\frac{d^{2}}{d X^{2}}-c^{2}\right) \widetilde{G}(X)=-\delta(X)$. All the amplitudes we study in this paper can be expanded in terms of (2.4). Hence this example is of special importance.

The advantage of working in position space is that now we can simply sum over all the contributions of the array of D-branes explicitly:

$$
\widetilde{G}_{\text {array }}(X)=\frac{\pi}{c} \sum_{n=-\infty}^{\infty} e^{-c\left|a\left(n+\frac{1}{2}\right)+X\right|} .
$$




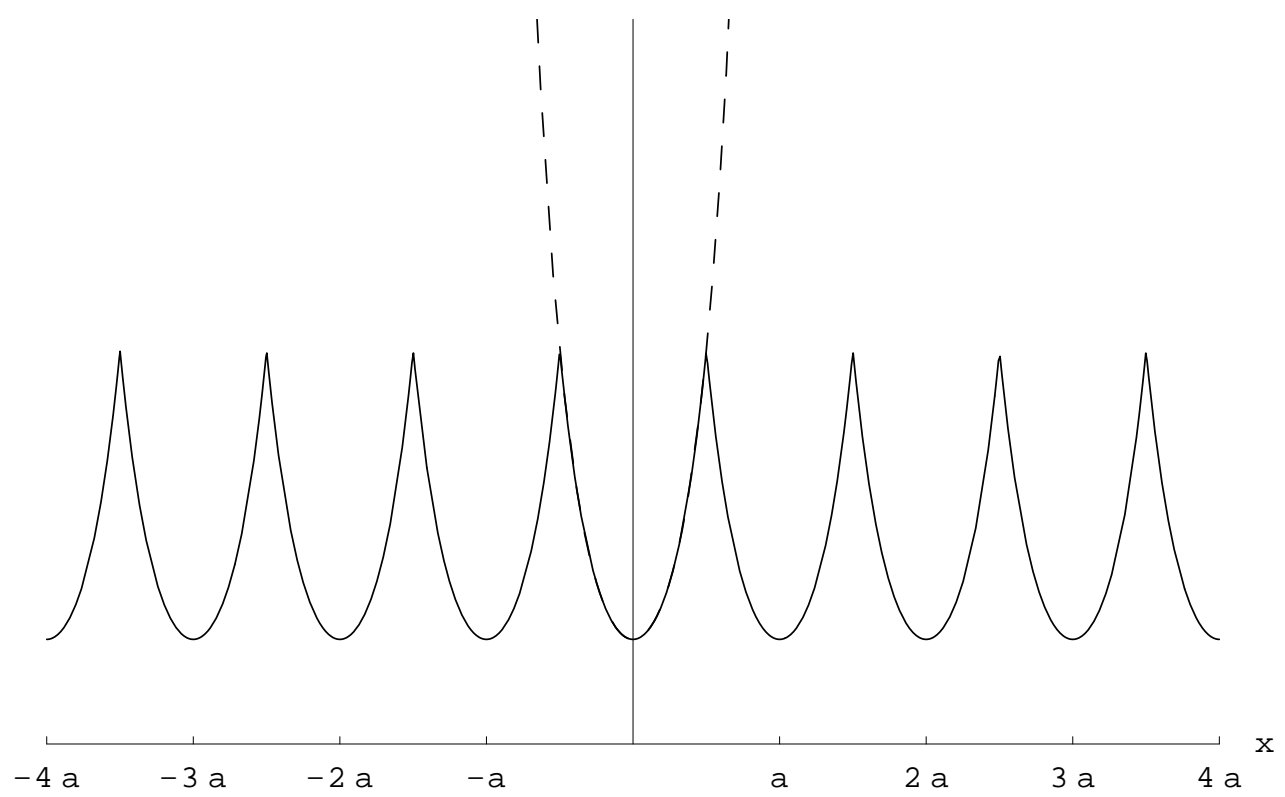

Figure 1: A graph of $\widetilde{G}_{\text {array }}(X)$, which has the interpretation of the field produced by an infinite array of $\delta$-function sources ('D-branes') located at $X=a\left(n+\frac{1}{2}\right)$. The dashed line represents the analytic continuation to $|X|>\frac{a}{2}$ of the branch around the origin.

This is a periodic function with period $a$ (see Fig.1), given in a neighborhood of $X=0$ by

$$
\widetilde{G}_{\text {array }}(X)=\frac{\pi \cosh (c X)}{c \sinh \left(\frac{c a}{2}\right)}, \quad|X| \leq \frac{a}{2} .
$$

Now we wish to Wick rotate. Of course, $\widetilde{G}_{\text {array }}(X)$ is not an analytic function, precisely because of the $\delta$-function sources located at $X=\left(n+\frac{1}{2}\right) a$. So we need to specify what we exactly mean by the analytic continuation $X \rightarrow-i X^{0}$. A natural prescription is to focus on the branch around the origin to find

$$
G_{\text {array }}\left(X^{0}\right)=\frac{\pi \cos \left(c X^{0}\right)}{c \sinh \left(\frac{c a}{2}\right)} .
$$

Fourier transforming (2.8) back to momentum space we find

$$
S(E, \ldots)=\frac{\pi}{2 c \sinh \left(\frac{c a}{2}\right)}(\delta(E-c)+\delta(E+c)) .
$$

As anticipated by the heuristic discussion in the previous subsection, while $\tilde{A}(P, \ldots)$ is non-zero for any real $P$, we find that $S(E, \ldots)$ has support only for those values of $E$ 
corresponding to singularities of $\tilde{A}(P, \ldots)$, namely $E=-i P= \pm c$. As we shall see in the context of string theory this will have the interpretation of a change in the dimension of the moduli space.

\subsection{General prescription}

In principle, all amplitudes considered in the paper could be expanded in terms of the example studied above. It would be nice however to have a general formula that gives $S(E, \ldots)$ in terms of $\widetilde{A}(P, \ldots)$. To this end consider, for generic $\widetilde{A}(P, \ldots)$,

$$
\widetilde{G}_{\text {array }}(X)=\int_{-\infty}^{\infty} d P e^{i P X} \sum_{n=-\infty}^{\infty}(-1)^{n} 2 \pi \delta(a P-2 \pi n) \widetilde{A}(P, \ldots) .
$$

In all cases that we study in the present paper $\widetilde{A}(P, \ldots)$ is an analytic function with poles or cuts only along the imaginary $P$ axis. Moreover $\widetilde{A}(P, \ldots)$ goes to zero for $|P| \rightarrow 0$ sufficiently fast to validate to following argument.

With the help of the residues theorem we can write

$$
\widetilde{G}_{\text {array }}(X)=\frac{1}{2 i} \oint_{\mathcal{C}} d P e^{i P X} \frac{\widetilde{A}(P, \ldots)}{\sin \left(\frac{a P}{2}\right)},
$$

where the contour $\mathcal{C}$ is depicted in Fig.2. By our analyticity assumptions on $\widetilde{A}(P, \ldots)$, the curve $\mathcal{C}$ can be deformed to $\tilde{\mathcal{C}}$ without crossing any singularities (see Fig.2) and without picking up any contributions from the two semi-circles at infinity (that are not shown in the figure). So we conclude, after Fourier transforming back to momentum space, that

$$
S(E)=F(E) \operatorname{Disc}_{E}[\widetilde{A}(i E)]
$$

where

$$
F(E)=\frac{1}{2 \sinh \left(\frac{a E}{2}\right)} .
$$

Here by $\operatorname{Disc}_{E}$ we mean the discontinuity with respect to $E$, namely

$$
\operatorname{Disc}_{E}[f(E)]=\frac{f(E+i \epsilon)-f(E-i \epsilon)}{i},
$$

so for example $\operatorname{Disc}_{E}(1 / E)=-2 \pi \delta(E)$. Let us check our master formula (2.12, 2.13) in the example studied in the previous subsection. From (2.4), $\widetilde{A}(i E)=1 /\left(-E^{2}+c^{2}\right)$; applying (2.12, 2.13) we immediately reproduce the result (2.9). 


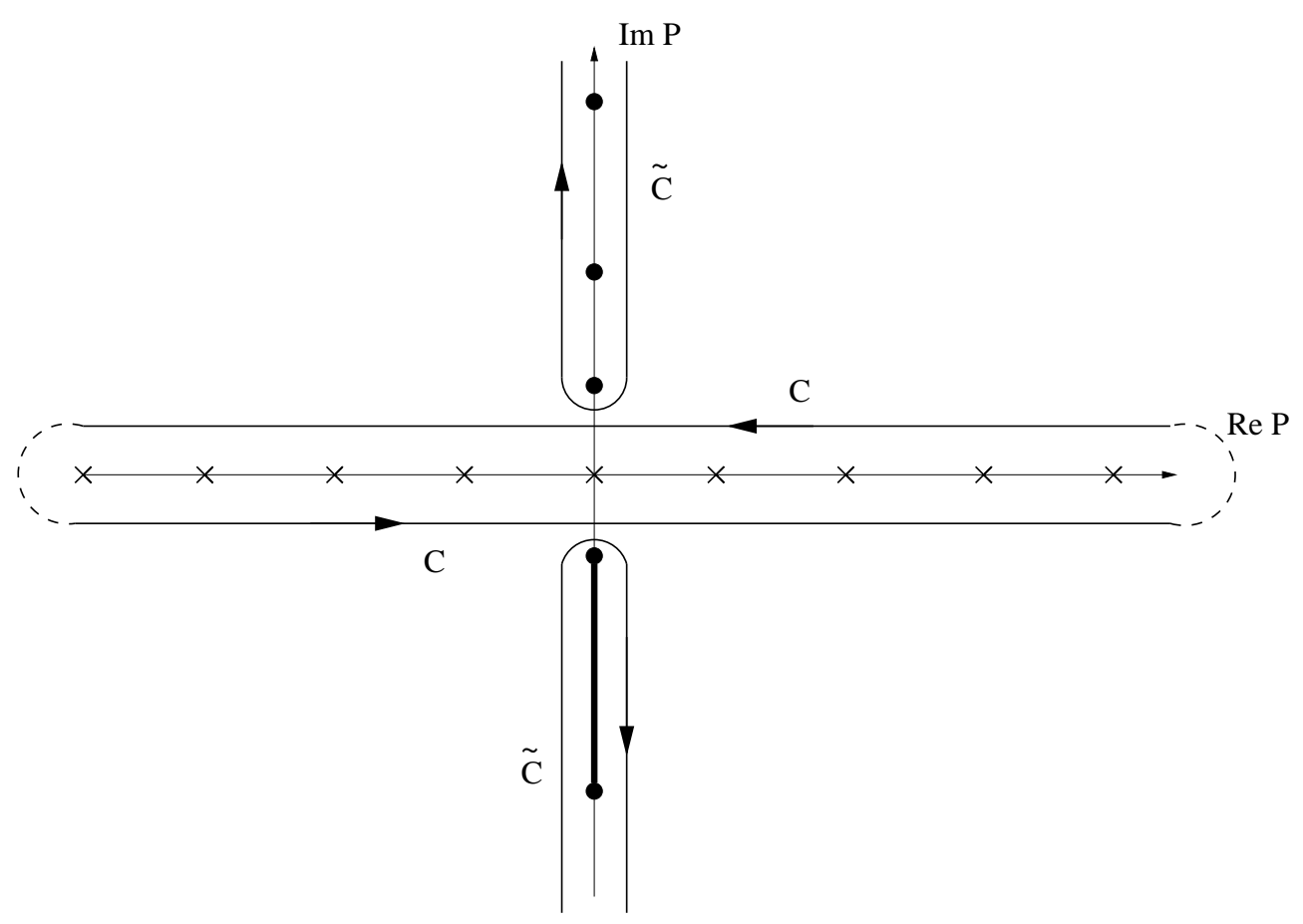

Figure 2: Integration contours in the complex $P$ plane. The zeros of $\sin (a P / 2)$ are denoted by the symbols ' $x$ ' along the real $P$ axis. The black dots represent possible poles of $\widetilde{A}$ and the thick line represents a possible cut. We assume that the only singularities of $\widetilde{A}$ are on the imaginary $P$ axis.

\section{Disk amplitudes}

To gain some insight into the meaning of the D-brane array at imaginary times (1.2), we now turn to a detailed analysis of disk scattering amplitudes of external closed strings. We start by considering the simplest possible case, namely the amplitude of two closed string tachyons. We first study this concrete example using standard methods, and then describe a more abstract point of view, that can be generalized easily to $m$-point functions involving arbitrary on-shell closed strings. A clear physical interpretation will emerge.

\subsection{Tachyon two-point amplitude}

The simplest non-trivial case that we consider is the disk amplitude with the insertion of two closed string tachyons. This example, which we are going to work out in full detail, contains already much of the essential physics.

To apply the prescription derived in the previous section, we need to evaluate the 
disk two-point function $\widetilde{A}\left(p_{1}, p_{2}\right)$ for a standard D-brane. We consider a $\mathrm{D}(p-1)$ brane with Dirichlet boundary conditions for $\widetilde{X}^{M}, M=0, \cdots, 25-p$ and Neumann boundary conditions for $\widetilde{X}^{m}, m=26-p, \cdots, 25$. We are using a notation that will be natural for the theory after the Wick rotation: $\widetilde{X}^{0}$ is a spatial coordinate that will become timelike after Wick rotation. In order to have a standard D-brane with Neumann conditions in time, we take one of the $\widetilde{X}^{m}$ to be timelike. So the Wick rotation is actually a double Wick rotation: It transforms $\widetilde{X}^{0}$ from spacelike to timelike and one of the parallel directions $\widetilde{X}^{m}$ from timelike to spacelike ${ }^{1}$. In practice, the amplitude $\widetilde{A}\left(p_{1}, p_{2}\right)$ will be written in terms of kinematic invariants, so these distinctions are of no much consequence.

It is convenient to break up the momenta into parallel and perpendicular directions,

$$
p^{\mu}=\left(p_{\perp}^{M}, p_{\|}^{m}\right),
$$

and define the kinematic invariants

$$
\begin{aligned}
& s=p_{1 \|}^{2}=p_{2 \|}^{2} \\
& t=\left(p_{1}+p_{2}\right)^{2}=\left(p_{1 \perp}+p_{2 \perp}\right)^{2} .
\end{aligned}
$$

In our conventions ${ }^{2} \alpha^{\prime}=1$, so that the on-shell condition for closed string tachyons is $p^{2}=4$.

The two-point disk amplitude has one modulus (four real coordinates minus three conformal Killing vectors). We work with a slightly unconventional parametrization of this moduli space that will be easy to generalize later when we turn to higher-point functions. Namely we shall fix the positions of the two vertex operators and integrate over the radius of the disk. We represent the disk as the complex domain $\mathcal{H}_{\rho}=\{z \in \mathbf{C},|z| \geq \rho\}$, that is we cut out a hole of radius $\rho$ from the complex plane. The measure for the modulus $\rho$ is

$$
\left(b_{0}+\bar{b}_{0}\right) \frac{d \rho}{\rho} \text {. }
$$

Using the standard doubling trick, a closed string vertex operator $V(p, z, \bar{z})$ is replaced by the two chiral insertions $V_{L}(p, z)$ and $V_{L}\left(p^{\prime}, \rho^{2} / \bar{z}\right)$, where $p^{\prime}=\left(-p_{\perp}, p_{\|}\right)$. For closed string

\footnotetext{
${ }^{1}$ The case $p=0$ is special, as we must choose one of the transverse directions $\widetilde{X}^{M}$ to be timelike.

${ }^{2}$ Moreover in writing a string amplitude $\widetilde{A}\left(p_{1}, \ldots p_{m}\right)$, we treat all momenta as incoming. Finally our convention for the Minkowski metric is 'mostly plus'.
} 
tachyons, $V(p, z, \bar{z})=c(z) \bar{c}(\bar{z}) \exp (i p X(z, \bar{z}))$ and $V_{L}(p, z)=c(z) \exp \left(i p X_{L}(z)\right)$. Fixing the tachyon vertex operators at $z=1$ and $z=\infty$, we have

$$
\widetilde{A}\left(p_{1}, p_{2}\right)=\frac{1}{2 \pi i} \int_{0}^{1} \frac{d \rho}{\rho} \oint_{|z|=\rho} d z\left\langle V_{L}\left(p_{1} ; \infty\right) V_{L}\left(p_{2} ; 1\right) b(z) V_{L}\left(p_{2}^{\prime} ; \rho^{2}\right) V_{L}\left(p_{1}^{\prime} ; 0\right)\right\rangle \text {, }
$$

where the symbol $\langle$,$\rangle denotes a CFT correlator on the plane. A short calculation gives$

$$
\widetilde{A}\left(p_{1}, p_{2}\right)=\int_{0}^{1} d \rho \rho^{t / 2-3}\left(1-\rho^{2}\right)^{s-2}=\frac{\Gamma(t / 4-1) \Gamma(s-1)}{2 \Gamma(t / 4+s-2)} .
$$

This is of course a standard result, with a familiar interpretation [29, 30]. The scattering amplitude of a closed string off a D-brane shows the usual 'dual' structure with poles both in the open and in the closed string channel. In the open string channel, the poles are located at $s=1,0,-1, \ldots$ and arise from expanding around $\rho=1$ where the vertex operators approach the boundary. In the closed string channel, we see poles at $t=$ $4,0,-4, \ldots$, arising from expanding around $\rho=0$ where the boundary shrinks to zero size.

We are now in a position to apply the prescription $(2.12)^{3}$. The discontinuity with respect to $E=E_{1}+E_{2}=-i\left(p_{1}^{0}+p_{2}^{0}\right)$ comes from the poles in the variable $t$, and so we find that

$$
S\left(p_{1}, p_{2}\right)=\frac{1}{2 \sinh \left(\frac{a|E|}{2}\right)} \sum_{k=0}^{\infty} f_{k}(s) \delta(t / 4-1+k),
$$

where

$$
f_{k}(s)=\frac{(-1)^{k} \Gamma(s-1)}{2 k ! \Gamma(s-k-1)}=\frac{(2-s)(3-s) \cdots(1+k-s)}{2 k !} .
$$

Several remarks are in order. First, all the contributions to $S$ come from the $\rho \rightarrow 0$ region of the moduli space. The sharpest way to see this is to introduce a cut-off $\epsilon \leq \rho \leq 1$. Then the amplitude $\widetilde{A}$ becomes analytic in $t$ since the poles in the closed string channel disappear. Applying (2.12) yields $S=0$ for any $\epsilon$. This vindicates the original intuition that the boundary state for the array of imaginary D-branes should correspond to a 'hole of zero size' (an extra puncture) in the worldsheet. This intuition will be made very precise below. Second, $S$ has no poles in $s$, the open string channel! The external closed strings do not couple to any on-shell open string degrees of freedom. Since there are no

\footnotetext{
${ }^{3}$ Notice that $\widetilde{A}\left(p_{1}, p_{2}\right)$ obeys our analyticity assumptions, indeed it is an analytic function of the total momentum $P^{0}$ with singularities (poles) only for imaginary $P^{0}$, and behaves as $1 /\left|P^{0}\right|^{2 s}$ for large $\left|P^{0}\right|$.
} 
D-brane sources in real time, this is as expected. We are describing a purely closed string background.

More precisely, (3.6) describes a sphere amplitude with the two tachyons insertions and an additional on-shell closed string state that involves excitation at all levels of the tower of massive modes. Indeed, the prefactor $f_{k}(s)$ is a polynomial of degree $2 k$ in $p_{\|}$, consistently with the fact that a closed string mode at level $k$ has up to $2 k$ Lorentz indices.

\subsection{Boundary state computation}

In order to secure this result, and to prepare for the generalization to higher-point amplitudes, we now repeat the computation in a more abstract language. We still write the amplitude in the domain $\mathcal{H}_{\rho}$, but instead of using the doubling trick, we represent the effect of the boundary at $|z|=\rho$ by the insertion of a boundary state $\left|\widetilde{\mathcal{B}}^{p-1}\right\rangle_{|z|=\rho}$. This is the full boundary state defining the $\mathrm{D}(p-1)$ brane located at $\widetilde{X}^{M}=0$.

A boundary state is a ghost number three state in the closed string Hilbert space, obeying among other things the conditions

$$
\left(Q_{B}+\bar{Q}_{B}\right)\left|\widetilde{\mathcal{B}}^{p-1}\right\rangle=0, \quad\left(b_{0}-\bar{b}_{0}\right)\left|\widetilde{\mathcal{B}}^{p-1}\right\rangle=0 .
$$

In radial quantization, a state at radius $\rho$ can be obtained from a state at radius $\rho=1$ by propagation in the closed string channel,

$$
\left|\widetilde{\mathcal{B}}^{p-1}\right\rangle_{|z|=\rho}=\rho^{L_{0}+\bar{L}_{0}}\left|\widetilde{\mathcal{B}}^{p-1}\right\rangle_{|z|=1}
$$

We can then write

$$
\widetilde{A}\left(p_{1}, p_{2}\right)=\int_{0}^{1} \frac{d \rho}{\rho}\left\langle\left|V\left(p_{1} ; \infty, \infty\right) V\left(p_{2} ; 1,1\right)\left(b_{0}+\bar{b}_{0}\right) \rho^{L_{0}+\bar{L}_{0}}\right| \widetilde{\mathcal{B}}^{p-1}\right\rangle_{|z|=1} .
$$

To proceed, we insert a complete set of states $\{|k, i\rangle\}$,

$\widetilde{A}\left(p_{1}, p_{2}\right)=\int d k \sum_{i} \int_{0}^{1} \frac{d \rho}{\rho}\left\langle\left|V\left(p_{1} ; \infty, \infty\right) V\left(p_{2} ; 1,1\right)\right| k, i\right\rangle \rho^{\left(\frac{k^{2}}{2}+2 l_{i}\right)}\left\langle k, i\left|\left(b_{0}+\bar{b}_{0}\right)\right| \widetilde{\mathcal{B}}^{p-1}\right\rangle_{|z|=1}$,

where $l_{i}$ is the level of the state $|k, i\rangle$, and we integrate over the modulus $\rho$ to get

$$
\widetilde{A}\left(p_{1}, p_{2}\right)=\int d k \sum_{i}\left\langle\left|V\left(p_{1} ; \infty, \infty\right) V\left(p_{2} ; 1,1\right)\right| k, i\right\rangle \frac{1}{\frac{k^{2}}{2}+2 l_{i}}\left\langle k, i\left|\left(b_{0}+\bar{b}_{0}\right)\right| \widetilde{\mathcal{B}}^{p-1}\right\rangle_{|z|=1} .
$$


This expression exhibits the decomposition of the amplitude into a source term from the boundary state, a closed string propagator, and the 3-point interaction vertex. If we now apply the master formula (2.12) we find

$$
S\left(p_{1}, p_{2}\right)=\left\langle\left|V\left(p_{1} ; \infty, \infty\right) V\left(p_{2} ; 1,1\right)\right| W\right\rangle,
$$

where ${ }^{4}$

$$
\begin{aligned}
|W\rangle & \equiv \int d k \sum_{i}|k, i\rangle \frac{\delta\left(k^{2} / 2+2 l(i)\right)}{2 \sinh \left(\frac{a|E|}{2}\right)}\left\langle k, i\left|\left(b_{0}+\bar{b}_{0}\right)\right| \mathcal{B}^{p-1}\right\rangle_{|z|=1} \\
& =\frac{\delta\left(L_{0}+\bar{L}_{0}\right)}{2 \sinh \left(\frac{a|E|}{2}\right)}\left(b_{0}+\bar{b}_{0}\right)\left|\mathcal{B}^{p-1}\right\rangle_{|z|=1} .
\end{aligned}
$$

We see that the interaction of the two tachyons with the imaginary array is captured by a ghost number two closed string state $|W\rangle$. Using (3.8) and $\left\{b_{0}, Q_{B}\right\}=L_{0}$, we have

$$
\left(Q_{B}+\bar{Q}_{B}\right)|W\rangle=0, \quad\left(b_{0}-\bar{b}_{0}\right)|W\rangle .
$$

These are precisely the physical-state conditions for closed strings. Moreover, we have the freedom to add to $|W\rangle$ BRST trivial states, that would decouple in the computation of the correlator (3.13). We recognize $|W\rangle$ as an element of the closed string cohomology. A more detailed discussion of this state and of its space-time interpretation will be carried out in section 4 .

Finally we can trade the state $|W\rangle$ with a vertex operator insertion at the origin, and re-write the amplitude (3.13) as a CFT correlator in the plane,

$$
S\left(p_{1}, p_{2}\right)=\left\langle V\left(p_{1} ; \infty, \infty\right) V\left(p_{2} ; 1,1\right) \mathcal{W}(0,0)\right\rangle .
$$

This is manifestly the scattering amplitude for three closed strings on the sphere! Having explicitly performed the integral over $\rho$ there are no remaining moduli, as it should be for a sphere three-point function.

Clearly this conclusion does not depend on the vertex operators $V\left(p_{i}, z, \bar{z}\right)$ being closed string tachyons. The analysis immediately generalizes to arbitrary physical closed string vertex operators: in the background of the imaginary array, a two-point function on the disk is equal to a three-point function on the sphere, with the on-shell vertex operator $\mathcal{W}$ as the extra insertion.

\footnotetext{
${ }^{4}$ Notice that in the formula below we drop the 'tilde' on the symbol for the boundary state: $\left|\mathcal{B}^{p-1}\right\rangle$ denotes the boundary state after double Wick rotation.
} 


\subsection{Higher-point disk amplitudes}

The computation of higher-point amplitudes is now a straightforward generalization. We still represent the disk as the complex domain $\mathcal{H}_{\rho}$ and describe the moduli space of $m$ closed strings on the disk by fixing the positions of two vertex operators, say $V_{1}$ at $z=\infty$ and $V_{2}$ at $z=1$, and varying the positions of the other $m-2$ insertions and the radius $\rho$ of the hole. More precisely, we vary the $m-2$ coordinates over the full complex plane, $\left\{z_{i} \in \mathbf{C}, i=3, \ldots m\right\}$, and for a given choice of the $\left\{z_{i}\right\}$ we vary the radius $\rho$ between 0 and the distance of the closest insertion, $0 \leq \rho \leq \rho_{0}=\min \left[\left|z_{i}\right|, i=1, \ldots m\right]$ (see Fig.3). This way we cover moduli space exactly once, as can be easily checked for example by mapping the above configuration to the the interior of the unit disk. We then have

$$
\begin{aligned}
& \widetilde{A}\left(p_{1}, \ldots, p_{n}\right)=\int d^{2} z_{3} \ldots d^{2} z_{m} \int_{0}^{\rho_{0}} \frac{d \rho}{\rho} \\
& \left\langle\left|\mathcal{R}\left\{V_{1}\left(p_{1} ; \infty, \infty\right) V_{2}\left(p_{2} ; 1,1\right) \ldots V_{m}\left(p_{m} ; z_{m}, \bar{z}_{m}\right)\right\}\left(b_{0}+\bar{b}_{0}\right) \rho^{L_{0}+\bar{L}_{0}}\right| \widetilde{\mathcal{B}}^{p-1}\right\rangle_{|z|=1},
\end{aligned}
$$

where $\mathcal{R}\{$.$\} denotes radial ordering. Inserting as before an intermediate complete set of$ states, and performing the $\rho$ integral, we find

$$
\begin{aligned}
& \widetilde{A}\left(p_{1}, \ldots, p_{n}\right)=\int d k \sum_{i} \int d^{2} z_{3} \ldots d^{2} z_{m} \\
& \left\langle\left|\mathcal{R}\left\{V_{1}\left(p_{1} ; \infty, \infty\right) V_{2}\left(p_{2} ; 1,1\right) \ldots V_{m}\left(p_{m} ; z_{m}, \bar{z}_{m}\right)\right\}\right| k, i\right\rangle \frac{\rho_{0}^{\frac{k^{2}}{2}+2 l_{i}}}{\frac{k^{2}}{2}+2 l_{i}}\left\langle k, i\left|\left(b_{0}+\bar{b}_{0}\right)\right| \widetilde{\mathcal{B}}^{p-1}\right\rangle_{|z|=1} .
\end{aligned}
$$

Now we extract the discontinuity with respect to the energy $E$. Clearly we get contributions from the poles in the propagators $\sim \frac{1}{k^{2} / 2+2 l i}$. A priori, the integrals over the coordinates $z_{i}$ may generate additional singularities. However it is not difficult to show that extra singularities can only arise when a (proper) subset of the vertex operators have an on-shell total momentum. We can define the amplitude by analytic continuation away from these singular points, and then disregard these singularities. This is reminiscent of the celebrated canceled propagator argument [31]. We can then write (see Fig.3)

$$
\left.S\left(p_{1}, \cdots, p_{m}\right)=\int d^{2} z_{3} \ldots d^{2} z_{m}\left\langle V_{1}\left(p_{1} ; \infty, \infty\right) V_{2}\left(p_{2} ; 1,1\right) \ldots V_{m}\left(p_{m} ; z_{m}, \bar{z}_{m}\right)\right\} \mathcal{W}(0,0)\right\rangle .
$$

\footnotetext{
${ }^{5}$ Notice that the dependence on $\rho_{0}$ drops because of the delta function $\delta\left(L_{0}+\bar{L}_{0}\right)$. In other terms, the $\rho$ integral localizes to $\rho \rightarrow 0$ and the upper limit of integration $\rho_{0}$ is immaterial.
} 


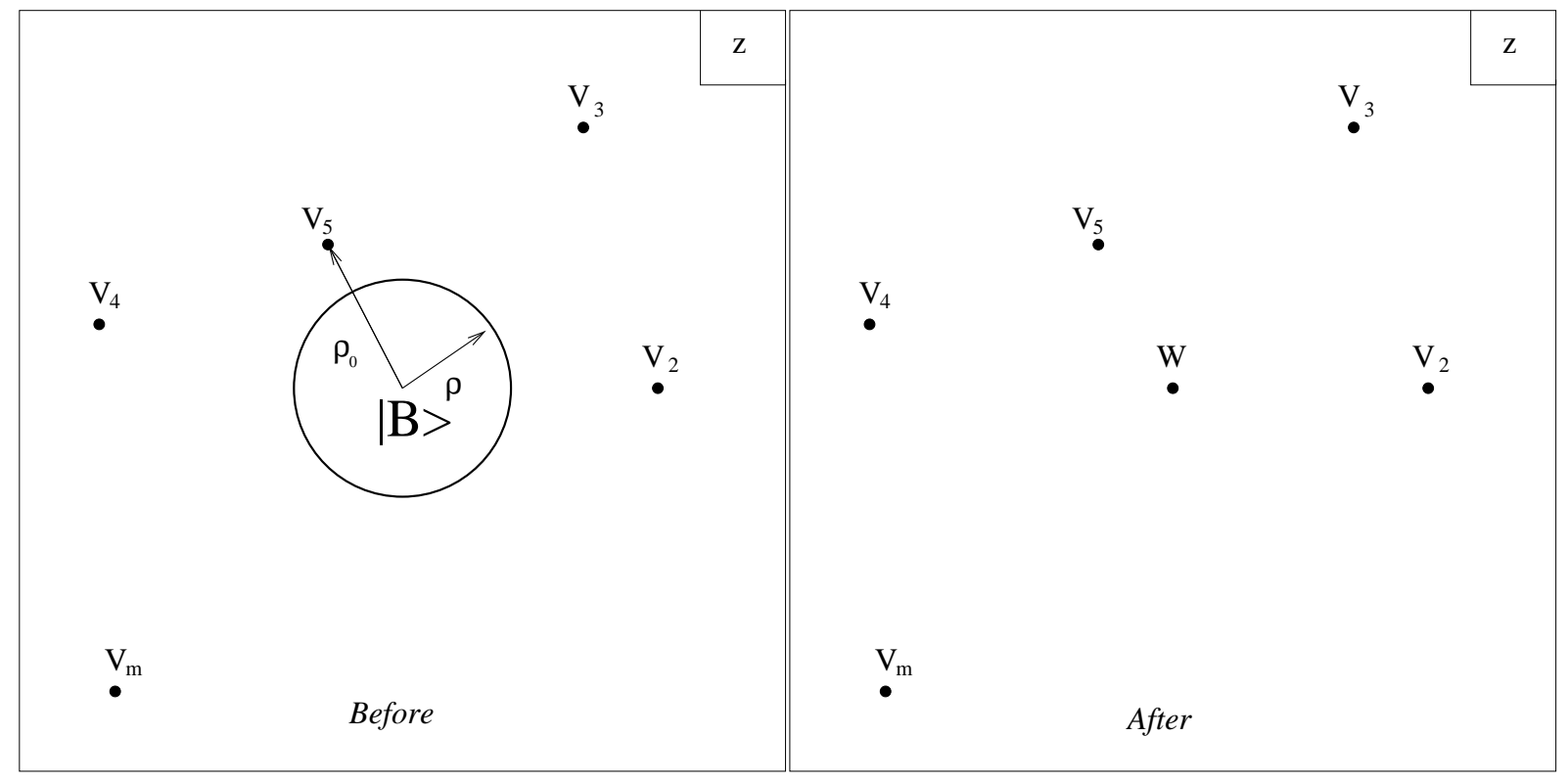

Figure 3: Before the double Wick rotation we have a standard disk amplitude. The disk can be viewed as the region $\mathcal{H}_{\rho}$, which is the complex plane with a hole of radius $\rho$. There are contributions to the scattering amplitude from all values of $\rho \leq \rho_{0}$, where $\rho_{0}$ is the distance of the closest puncture. After the double Wick rotation the only contribution is coming from $\rho=0$. The hole shrinks to a point leaving behind an extra puncture $\mathcal{W}$ inserted at the origin.

The coordinates $z_{i}, i=3, \ldots m$, are integrated over the full complex plane, so this is the string theory amplitude for $m+1$ insertions on the sphere. We can check that the counting of moduli is consistent with this result. We started with $2 m-3$ moduli for a disk with $m$ closed punctures and performed an explicit integration over $\rho$. This gives $2 m-4=2(m+1)-6$, which is the number of moduli for a sphere with $m+1$ punctures.

\subsection{Interpretation}

Let us summarize what we have learned. Disk amplitudes for $\mathrm{m}$ external closed strings off the D-brane array at the imaginary times $X^{0}=i(n+1 / 2)$ a are completely equivalent to $\mathrm{m}+1$-point amplitudes on the sphere, with the extra closed string insertion

$$
g_{s} \int d^{2} z \mathcal{W}(z, \bar{z})
$$

where $\mathcal{W}$ is given by (3.14). The explicit factor of $g_{s}$, which was omitted in the previous formulae, comes from the relative normalization of disk amplitudes to standard sphere 
amplitudes. Unlike the case of an ordinary brane, we are finding that for the imaginary array disk amplitudes give a purely closed correction (of order $g_{s}$ ) to the background. There are no D-brane sources in real time, only closed strings satisfying the homogeneous wave equation.

The absence of sources in real time can also be deduced from the fact that all one-point functions on the disk are trivially zero in our prescription. This is consistent with the computations in [3], where the stress tensor (related to the graviton one-point function) was found to vanish in the BCFT (1.1) for $\lambda=\frac{1}{2}$. There are two equivalent ways to see this in our language. The one-point function on the disk is a smooth function of the total energy $E$, so there is no discontinuity in $E$. Alternatively, the discussion above implies that a disk one-point function is equal to a sphere two-point function with the extra insertion of $\mathcal{W}$; but sphere two-point functions are zero because of the infinite volume of the unfixed moduli.

Since disk amplitudes provide the first order correction to the background, it is very natural to expect that amplitudes with multiple boundaries give the necessary higherorder corrections. A genus zero amplitude with $m$ external closed strings and $b$ boundaries, is expected to become after Wick rotation a sphere amplitude with $m+b$ punctures, with $b$ insertions of $g_{s} \int d^{2} z \mathcal{W}$. Notice that since the boundaries are indistinguishable, the sum over boundaries exponentiates to the insertion of $\exp \left(\int d^{2} z g_{s} \mathcal{W}\right)$, which has the interpretation of a coherent state of closed strings. One may investigate this issue rigorously by representing the effect of the boundaries using boundary states, and decomposing the moduli space of a surface with $b$ boundaries and $m$ closed string punctures in terms of closed string vertices and propagators [32. The moduli space integration should localize to the region where each boundary shrinks to an extra puncture. A priori, one may also expect that regions of moduli space where zero-size boundaries collide could provide additional contributions. For example one may expect that an annulus amplitude would reduce to a sphere amplitude with two insertions of $g_{s} \mathcal{W}$, plus a sphere amplitude with a single insertion of a new operator $g_{s}^{2} \mathcal{W}_{1}$ coming from the shrinking of both boundaries to the same point. This issue should be investigated further. 


\section{The closed string state}

In this section we study the physical properties of $|W\rangle$ in more detail and make contact with [1] and [12]. Our starting point is the boundary state $\left|\mathcal{B}^{p-1}\right\rangle$, which is obtained from double Wick rotation of $\left|\widetilde{\mathcal{B}}^{p-1}\right\rangle$. So $\left|\mathcal{B}^{p-1}\right\rangle$ is associated with a $\mathrm{D}(p-1)$ brane located at $X^{M}=0$, in a space with metric $\left(\eta_{M N}, \delta_{m n}\right)$. One has

$$
\left|\mathcal{B}^{p-1}\right\rangle=\mathcal{N} \delta\left(X^{M}\right) \exp \left(-\sum_{n=1}^{\infty} a_{n}^{\mu \dagger} S_{\mu \nu} \tilde{a}_{n}^{\nu \dagger}\right)|0 ; k=0\rangle
$$

$\mathcal{N}$ is a normalization constant that can be found for example in [33], and

$$
S_{M N}=-\eta_{M N}, \quad S_{m n}=\delta_{m n}
$$

With the help of (3.14) we can expand $|W\rangle$ in terms of closed string physical states,

$|W\rangle=\mathcal{N} c_{1} \bar{c}_{1}|0\rangle_{g h} \otimes \int \frac{d k_{\perp}^{25-p}}{(2 \pi)^{25-p}} \frac{1}{2|E|} \frac{1}{2 \sinh \left(\frac{a|E|}{2}\right)}\left(\left|k^{2}=4\right\rangle-S_{\mu \nu} \partial X^{\mu} \bar{\partial} X^{\nu}\left|k^{2}=0\right\rangle+\cdots\right)$,

where the dots indicate higher massive modes. The first term is the closed string tachyon, which is an artifact of the bosonic string and is standard practice to ignore. Below we first discuss the massless modes and then consider the massive modes.

\subsection{Massless sector}

In order to read off the dilaton and gravity wave profile from the second term we have to undo their mixing. The unmixed dilaton and graviton (in the Einstein frame) take the form (see e.g. [33])

$$
h_{\mu \nu}=S_{\mu \nu}-\frac{S \cdot \epsilon^{(\phi)}}{\eta \cdot \epsilon^{(\phi)}} \eta_{\mu \nu}, \quad \phi=S \cdot \epsilon^{(\phi)}
$$

where

$$
\epsilon^{(\phi)}=\frac{1}{2}\left(\eta_{\mu \nu}-k_{\mu} l_{\nu}-k_{\nu} l_{\mu}\right), \quad k \cdot l=1, \quad l^{2}=0 .
$$

Let us first look at the case $p=0$ (an array of imaginary $\mathrm{D}(-1)$ branes). One finds that the Einstein metric is completely flat as all the expectation values of $h_{\mu \nu}$ vanish. This means that the part of the leading term in the ADM mass, which scales like $1 / g_{s}$, vanishes (see also [12] $)^{6}$. The dilaton, on the other hand, does not vanish. So for $p=0$, the massless

\footnotetext{
${ }^{6}$ This is consistent with Sen's observation [3] that the stress tensor vanishes for the $\lambda=\frac{1}{2}$ BCFT (1.1).
} 
fields in $|W\rangle$ consist of a spherically symmetric dilaton wave $\phi\left(r, X^{0}\right)$ in $25+1$ dimensions, whose energy is of order $O\left(g_{s}^{0}\right)=O(1)$.

For $p>0$ the 26-dimensional metric in the Einstein frame is non-trivial. However the fields profiles are translationally invariant in the $p$ longitudinal directions $X^{m}$, and to read off the ADM mass we are instructed to dimensionally reduce to the $26-p$ transverse dimensions. One finds that in the $26-p$-dimensional Einstein frame the metric is zero, and we have again only a spherically-symmetric dilaton wave. We conclude that to order $1 / g_{s}$ the mass vanishes for general $p$.

The space-time profile of this dilaton wave is quite interesting [11. ${ }^{7}$ For fixed radius $r=\sqrt{X^{M} X^{M}}$, the field decays exponentially fast as $X^{0} \rightarrow \pm \infty$. For fixed $X^{0}$, the field decays as $1 / r^{23-p}$ as $r \rightarrow \infty$, just like the fields produced by an ordinary $\mathrm{D} p$-brane, but with a different numerical coefficient. (These two asymptotic behaviors match in a region of thickness of the order of $a$ around the light-cone $X^{0}= \pm r$ ). To be precise, up to an overall numerical constant that will not be relevant for the discussion below, the leading asymptotic behavior as $r \rightarrow \infty$ of the various fields is, in the 26-dimensional Einstein frame,

$$
\begin{aligned}
& h_{00} \rightarrow \frac{(1+K)(d-3)-2 p}{d-2} \frac{1}{r^{23-p}} \\
& h_{m n} \rightarrow \frac{\delta_{m n}}{2}\left(\frac{2 d-2 p-5-K}{d-2}\right) \frac{1}{r^{23-p}}, \\
& h_{M N} \rightarrow \frac{\delta_{M N}}{2}\left(\frac{2 p+1+K}{d-2}\right) \frac{1}{r^{23-p}} \\
& \phi \rightarrow \frac{d-2 p-3-K}{4} \frac{1}{r^{23-p}}
\end{aligned}
$$

Here $d=26$ and the parameter $K$ is set to 1 for the standard D $p$-brane and is set to -1 for the background $|W\rangle$.

An exercise one can now do is to compute the force acting on a probe D0-brane in this dilaton wave background [11. One has to be a bit careful here with the exact meaning of the 'force' between the brane and the wave since this is not a static set-up. The 'force' that can be computed using (4.5) for $K=-1$ is acting during a finite time interval $-T \leq X^{0} \leq T$ at a distance $r$ in the limit $\frac{T}{r} \rightarrow 0$. In the Einstein frame in 26 dimensions

\footnotetext{
${ }^{7}$ From the discussion in section 6 of [1] it is not obvious that eq.(6.27) in that section describes just a dilaton wave. However, this can be verified with the help of eq. 4.3) above.
} 
the DBI action for the D0 brane takes the form

$$
\int d X^{0} e^{-\frac{11}{12} \phi} \sqrt{g_{00}}
$$

From this and (4.5) with $p=0$ we can deduce that the ratio between the force acted upon the D0 by a standard brane and the 'force' acted on it by the dilatonic wave in $|W\rangle$ is

$$
\frac{F_{\text {standard }}}{F_{W}}=\frac{\frac{11}{12} \phi(K=1)+\frac{1}{2} h_{00}(K=1)}{\frac{11}{12} \phi(K=-1)+\frac{1}{2} h_{00}(K=-1)}=\frac{12}{11} .
$$

This is in agreement with [1] where this ratio was calculated in a different way.

\subsection{Massive modes}

The massive closed string states in $|W\rangle$ obey the dispersion relation $E^{2}=k_{\perp}^{2}+m^{2}$, with $m^{2}=4(n-1)$. For $n>1$, their field profile (proportional to $1 / \sinh (a|E| / 2)$ ) is strongly peaked at energies $|E|-m \leq 1 / a$. This means that for any $a \geq 2 \pi$, the states are with good approximation non-relativistic already at level $n=2$, with the approximation improving at higher levels. Their fields at $X^{0}=0$ are Gaussians of the form

$$
\delta\left(k_{\|}\right) \exp \left(-\frac{m a}{2}\right) \exp \left(-\frac{a k_{\perp}^{2}}{4 m}\right)
$$

that is, the closed string modes occupy the directions $X^{m}$ and are localized at $X^{M}=0$ $(M \neq 0)$ with a width of order $\sqrt{a / m}$ in the transverse directions. The time evolution of these field profiles follows non-relativistic Schrödinger equation, so their width scales as $\left|X^{0}\right| / \sqrt{a m}$ for large times. Interestingly, the massive modes behave as non-relativistic matter located at the would-be position of the brane. This conclusion was also reached in 12.

Let us now compute the normalization of $|W\rangle$ and its the space-time energy, following $[12]^{8}$. The normalization has the interpretation of (the expectation value of) the total number of particles $\bar{n}$ in the background. One has

$$
\begin{aligned}
\bar{n}=\left\langle\mathcal{W}\left|c_{0} \bar{c}_{0}\right| \mathcal{W}\right\rangle & =\mathcal{N}^{2} \sum_{n=0}^{\infty} d_{n} \int \frac{d k_{\perp}^{25-p}}{(2 \pi)^{25-p}} \frac{1}{2|E|} \frac{1}{4 \sinh ^{2}\left(\frac{a E}{2}\right)}, \\
\bar{E} & =\mathcal{N}^{2} \sum_{n=0}^{\infty} d_{n} \int \frac{d k_{\perp}^{25-p}}{(2 \pi)^{25-p}} \frac{1}{2|E|} \frac{|E|}{4 \sinh ^{2}\left(\frac{a E}{2}\right)}
\end{aligned}
$$

${ }^{8}$ Equs. (4.9) and (4.11) below were not obtained independently of [12]. 
where $d_{n}$ can be computed from the generating function

$$
\sum_{n=0}^{\infty} d_{n} w^{n}=f(w)^{-24}, \quad f(w)=\prod_{m=1}^{\infty}\left(1-w^{n}\right) .
$$

The asymptotic behavior of $d_{n}$ for large $n$ is 34] $d_{n} \sim n^{-27 / 4} e^{4 \pi \sqrt{n}}$. It is easy to see that for $a>2 \pi$ the exponential suppression from $1 / \sinh ^{2}\left(\frac{a|E|}{2}\right)$ wins over the exponential growth of states, and both $\bar{n}$ and $\bar{E}$ are perfectly finite. On the other hand, in the limit $a \rightarrow 2 \pi$ we get

$$
\bar{E} \sim \sum_{n=0}^{\infty} \frac{e^{-2(a-2 \pi) \sqrt{n}}}{n^{p / 4}} \sim(a-2 \pi)^{p / 2-1} .
$$

For $a=2 \pi$ the expectation value of the energy diverges for $p=0,1,2$. Naively, for $p>2$ the energy is finite. However, for any $p$ the expectation values of powers $\left\langle E^{k}\right\rangle$ will eventually diverge for sufficiently high $k$ [12, and hence for $a=2 \pi$ the uncertainty in the energy $^{9}$ is infinite for any $p$.

This divergence has a very natural physical interpretation. As reviewed in the introduction and further discussed in section 8 below, for $a=2 \pi$ the background of imaginary branes admits an infinitesimal deformation that introduces a D-brane source in real time, and we should then expect that this background also has an energy of order $1 / g_{s}$. Since we are computing this energy in the limit $g_{s} \rightarrow 0$ in a perturbative expansion $\bar{E}=\sum_{n=0}\left(g_{s}\right)^{n} E^{(n)}$, it is natural to find that leading term $E^{(0)}=\infty$. At finite $g_{s}$ this divergence should be regulated in such a way that $\bar{E} \sim 1 / g_{s}$.

Before discussing a heuristic mechanism that supports this expectation, we would like to point out that for $a=2 \pi$ the state $|W\rangle$ has all the features to be identified with 'tachyon matter' [3, 4]. Indeed for $a=2 \pi$ the energy is stored in very massive closed strings modes that behave like non-relativistic matter strongly localized along the directions $X^{m}$. This is the closed string dual of Sen's discussion on 'tachyon matter' in the context of open string field theory; for large times $\left|X^{0}\right| \rightarrow \infty$ the classical open string solution corresponding to the BCFT (1.1) approaches, for all $\lambda$, an open string configuration with zero pressure and with all the energy localized along the $X^{m}$ directions ${ }^{10}$.

\footnotetext{
${ }^{9} \mathrm{~A}$ similar behavior is found for $\bar{n}$, which is logarithmically divergent for $p=0$ and finite for $p>0$, but with higher moments diverging for any $p$.

${ }^{10}$ More precisely, as shown in [12] and further elaborated in section 8 of this paper, the limit $X^{0} \rightarrow \infty$ of (1.1) corresponds for all $\lambda$ (up to a trivial time translation) to the outgoing $\left(X^{0}>0\right)$ part of our state $|W\rangle$, and symmetrically $X^{0} \rightarrow-\infty$ of (1.1) corresponds to the incoming $\left(X^{0}<0\right)$ part of $|W\rangle$.
} 
It is very tempting to suspect that at finite $g_{s}$ the distance $a$ is renormalized to an effective value

$$
a_{\text {eff }}=2 \pi+\varepsilon \quad \text { where } \varepsilon=\gamma g_{s}^{b},
$$

where both $b$ and $\gamma$ are positive numbers of order one. This would make the energy finite. Restricting in the following to $p=0^{11}$, we find from (4.11)

$$
\bar{E} \sim \frac{1}{\varepsilon}
$$

So to obtain the expected scaling $\sim 1 / g_{s}$ the parameter $b$ has to be equal to one. An argument why this is plausible was given in [12] from the point of view of open string field theory. Here we provide an alternative heuristic argument from the closed string channel, that justifies why $b=1$ and also why $\gamma>0$.

Let us think about this issue from the point of view of the array before Wick rotation. When $g_{s}=0$ the distance between the branes is $2 \pi$. When $g_{s}$ is turned on the branes will slightly curve space-time due to their mass so that the distance between them is no longer $2 \pi$. Actually since their mass is $\sim 1 / g_{s}$ and the Newton constant scales like $g_{s}^{2}$ the back-reaction is of order $g_{s}$ and so the proper distance between the branes is indeed as in (4.12). $\gamma$ is positive simply because the metric components in the transverse direction to the brane scale like

$$
g_{\perp} \sim 1+g_{s} \frac{\tilde{\gamma}}{r^{23-p}}
$$

with $\tilde{\gamma}>0$.

It is interesting to check if this argument generalizes when we put $N$ rather than just one brane at each site. First, the back-reaction of the metric scales like $g_{s} N$ which means that now $\varepsilon \sim g_{s} N$. Combining this with (4.13) and with the fact that $|W\rangle$ gets multiplied by a factor of $N$ we find for the total energy

$$
\bar{E}_{N} \sim \frac{N^{2}}{\varepsilon} \sim \frac{N}{g_{s}},
$$

which is the correct scaling for the tension of $N$ D-branes.

Clearly this heuristic reasoning is not powerful enough to fix $\gamma$ in (4.12). One obvious reason is that the distance between the branes is of the order of the string scale and hence

\footnotetext{
${ }^{11}$ For $p>0$ (4.11) cannot be trusted as the higher moments of the energy $\left(\left\langle E^{k}\right\rangle-\langle E\rangle^{k}\right)^{1 / k}$ have a worse degree of divergence than the mean value $\bar{E}=\langle E\rangle$.
} 
the gravity approximation should not be trusted. A closely related point is the following. Suppose that we could somehow fix $\gamma$ and that we found the correct D-brane mass. If $\gamma$ was just a fixed number then it is easy to see that the uncertainty in the D-brane mass would be of the same order as the mass itself, which is of course not the case for D-branes at weak coupling. This seems to suggest that $\gamma$ should not be viewed as a constant but rather as a fluctuating field.

\section{On open and closed string moduli}

In section 3 we showed that scattering amplitudes off the imaginary array do not have any open string poles. This means that there are no propagating open strings degrees of freedom, consistently with the fact that there are no branes in real time. However there still is a discrete set of on-shell open string vertex operators, which demand an interpretation. In this section we argue that they are dual to deformations of the closed string state $|W\rangle$.

For simplicity let us start by considering the case $p=0$, the array of $\mathrm{D}(-1)$ branes at imaginary times $X^{0}=i\left(n+\frac{1}{2}\right) a$. The open string spectrum should be read off from the theory before double Wick rotation, where we have an array of $\mathrm{D}(-1)$ branes at the spatial locations $\widetilde{X}^{0}=\left(n+\frac{1}{2}\right) a$. For generic ${ }^{12}$ distance $a$, the only matter primaries of dimension one are $\widetilde{V}_{\mu}^{(n)}=\partial \widetilde{X}_{\mu}^{(n)}$, where $\mu$ are space-time indices and $n \in \mathbf{Z}$ labels the position of the $\mathrm{D}(-1)$ brane. In the double-Wick rotated theory the physical open string states are just the same, up to trivial relabeling. So the most general state in the open string cohomology of the theory of $\mathrm{D}(-1)$ branes at imaginary times can be written as

$$
|f\rangle=\sum_{\mu=0}^{25} \sum_{n=-\infty}^{\infty} f_{(n)}^{\mu} \partial X_{\mu}^{(n)}(0) c_{1}|0\rangle .
$$

Clearly these are the exactly marginal open string deformations that correspond to moving the positions of the $\mathrm{D}(-1)$ branes.

Next we can consider disk amplitudes $S\left(p_{1} \ldots p_{m} ; f\right)$ for $m$ closed strings scattering off the imaginary array, with one additional insertion of (15.1) on the boundary of the disk. Without any open strings puncture, $S\left(p_{1}, \ldots p_{m}\right)$ was shown in section 3 to be a sphere amplitude with an extra insertion of the closed string state $|W\rangle$. How does the addition of $|f\rangle$ change this conclusion?

\footnotetext{
${ }^{12}$ The case $a=2 \pi$ is of course special and will be discussed in section 8 .
} 
To get some insight, consider first the case $f_{(n)}^{\mu}=f^{\mu}$ for all $n$. This deformation is simply a Goldstone mode associated with a rigid translation $X_{\mu} \rightarrow X_{\mu}+f_{\mu}$ of the whole array. It is clear that in this simple case $S\left(p_{1} \ldots p_{m} ; f\right)$ is obtained from $S\left(p_{1} \ldots p_{m} ; f\right)$ by replacing $|W\rangle$ with its infinitesimal translation, $|W\rangle \rightarrow f^{\mu} \partial_{\mu}|W\rangle$. In this special example the open string insertion $|f\rangle$ corresponds to a symmetry of the vacuum broken by the closed string background $|W\rangle$.

In the more general case (5.1), it is not difficult to show ${ }^{13}$ that $S\left(p_{1} \ldots p_{m} ; f\right)$ will still be a sphere amplitude with $m+1$ punctures, where the extra closed puncture is now $\delta_{f} \mathcal{W}$, the infinitesimal deformation of $\mathcal{W}$ obtained by displacing the branes according to $|f\rangle$. Generically this deformation will change the total energy of the closed string state. The intuitive picture is that the branes act as sources for the closed string fields. Since these sources are not in real time, the resulting closed string fields are homogeneous solutions of the wave equation, and we have a purely closed background. Changing the positions of the branes changes the details of the closed string field profiles. Thus the open string moduli (5.1) are re-interpreted as deformations of the closed string background. In the next section this picture is made precise.

There is one important restriction on the allowed motions of the branes: The resulting closed string fields must be real. This imposes certain constraints on the moduli $f_{(n)}^{\mu}$. Starting from the array at $X^{0}=i n\left(a+\frac{1}{2}\right)$, reality of the closed string fields demands that the $\mathrm{D}(-1)$ branes be moved in pairs, that is, the $k$-th $\mathrm{D}(-1)$ brane at $X^{0}=i a\left(k+\frac{1}{2}\right)$, $k>0$, together with its mirror partner at $X^{0}=i a\left(-k+\frac{1}{2}\right)$. The reality condition is

$$
f_{(k)}^{\mu}=\left(f_{(-k)}^{\mu}\right)^{*} \quad \forall k \in \mathbf{N}
$$

where $*$ denotes complex conjugation. As long as we refrain from considering branes with complex spatial coordinates $X^{i}, i=1, \ldots 25$, we should keep the spatial moduli $f_{(k)}^{i}$ real, and then (5.2) implies $f_{(k)}^{i}=f_{(-k)}^{i}$. For the time coordinate $X^{0}$ on the other hand, there are two possible motions for a given pair of branes, as illustrated in Fig. $4^{14}$.

The reality condition can be simply rephrased by saying that the D-brane configuration must be symmetric under reflection with respect to real time axis. A simple way to see

\footnotetext{
${ }^{13}$ We cannot directly apply the prescription of section 2 . However, the relevant disk amplitude is simple enough that can be evaluated directly in the theory of the spatial array $\widetilde{X}^{0}=a\left(n+\frac{1}{2}\right)$, and then Wick rotated.

${ }^{14}$ Interestingly, for a pair of branes before the double Wick rotation there are also two allowed exactly marginal motions along $X=-i X^{0}$, but of course they are the independent translations of each brane along the real $X$ axis.
} 


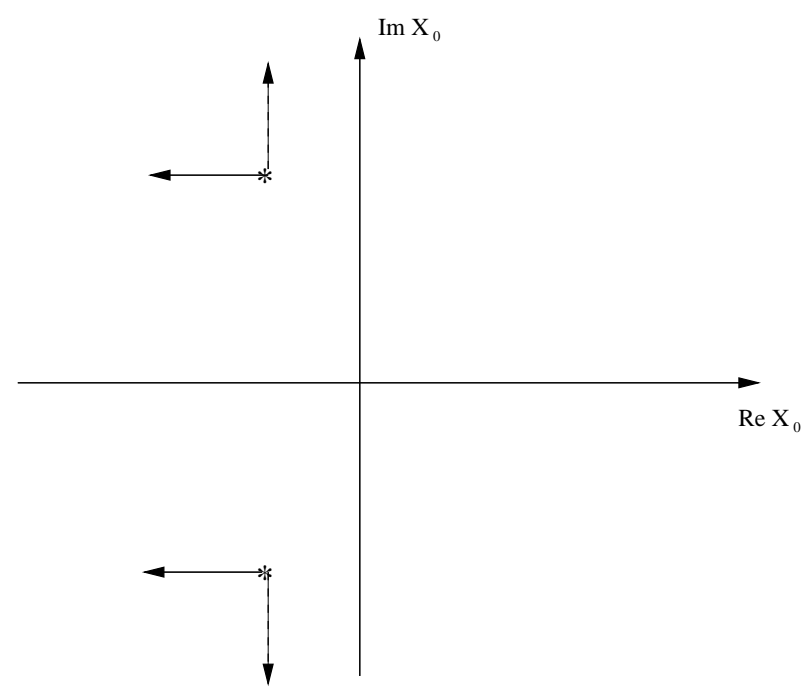

Figure 4: The two possible motion modes of a pair of D-branes in the complex $X^{0}$ plane.

this constraint is to focus for example on the massless closed string fields. The field produced by some $\delta$-function sources located at the origin in space and at times $X^{0}=Y_{k}^{0}$ is schematically [1]

$$
\phi\left(X^{\mu}\right) \sim \sum_{k} \frac{1}{\left[-\left(X^{0}-Y_{k}^{0}\right)^{2}+\left(X^{i}\right)^{2}\right]^{\sigma}}
$$

where the power $\sigma$ depends on the number of transverse directions. We are interested in measuring the field $\phi\left(X^{\mu}\right)$ for real values of its arguments $X^{\mu}$. It follows that for $\phi\left(X^{\mu}\right)$ to be real, the locations of the sources $\left\{Y_{k}^{0}\right\}$ must either be real or come in complex conjugate pairs. In the next section we write down the prescription to obtain the closed string background associated with an arbitrary configuration of imaginary branes, and it will be apparent that the same reality condition is valid in full generality.

For $p>0$ there are additional on-shell open strings since the open string tachyon can be put on-shell provided $p^{m} p^{m}=1$, where $p^{m}$ is the momentum along the Neumann directions. These are precisely the exactly marginal deformations studied in the original work by Callan et al. 25]. For example the deformation $\lambda^{\prime} \cos \left(X^{m}\right)$, where $X^{m}$ is one of the directions along the brane, continuously interpolates between Neumann and Dirichlet boundary conditions for the coordinate $X^{m}$. At the critical value $\lambda^{\prime}=\frac{1}{2}$, we get an array of $\mathrm{D}(p-2)$ branes localized at $X^{m}=2 \pi\left(n+\frac{1}{2}\right) i$. The deformed boundary state is known for all values of $\lambda^{\prime}$ [25] and we can easily apply our prescription (3.14) to compute $\left|W\left(\lambda^{\prime}\right)\right\rangle$. As $\lambda^{\prime}$ varies, these states are a family of purely closed string backgrounds. An open string deformation, in this case $\cos \left(X^{m}\right)$, is then again re-interpreted as a closed 
string deformation.

\section{A more general set-up}

One important conclusion from the discussion in the previous section is that there is nothing fundamentally special about the array of D-branes at $X^{0}=i\left(n+\frac{1}{2}\right) a$. Exactly marginal open string deformations allow to move the positions of the branes in the complex $X^{0}$ plane, with the only constraints coming from the reality condition. By sending off most of the branes to large imaginary time, we can also consider configurations with a finite number of branes.

The simplest configuration consists of only one pair of branes at $X^{0}= \pm i \beta$ (with $\beta$ real, $\beta>0$ ). As in section 2.2, we start with the pair in Euclidean space at $X= \pm \beta$. The analog of (2.7) is

$$
\widetilde{G}_{\text {pair }}(X)=\frac{2 \pi}{c} e^{-\beta c} \cosh (c X), \quad|X| \leq \beta .
$$

Notice that the only change relative to (2.7) is in the prefactor. This was foreordained since in a finite neighborhood of $X=0$ both $\widetilde{G}_{\text {pair }}(X)$ and $\widetilde{G}_{\text {array }}(X)$ obey homogeneous wave equation $\left(\frac{d}{d X^{2}}-c^{2}\right) \widetilde{G}=0$ and are even under $X \rightarrow-X$. Wick rotation then gives the usual sourceless solution $\sim \cos \left(c X^{0}\right)$. In Fourier transform

$$
S_{\text {pair }}(E, \ldots)=\frac{\pi}{2 c} e^{-\beta c}(\delta(E-c)+\delta(E+c))
$$

which we can write

$$
S_{\text {pair }}(E, \ldots)=F_{\text {pair }}(E) \operatorname{Disc}_{E}[\widetilde{A}(i E)]
$$

where

$$
F_{\text {pair }}(E)=\operatorname{sign}(E) e^{-\beta|E|} .
$$

This result should be compared with (2.9), (2.12), (2.13).

This formula can be generalized further by displacing the D-brane pair along real time, that is at $X^{0}=\alpha \pm i \beta$. In this case $F_{\text {pair }}(E) \rightarrow \operatorname{sign}(E) e^{-\beta|E|+i \alpha E}$. By linear superposition, an arbitrary configuration of $M$ D-brane pairs at positions $X^{0}=\alpha_{k} \pm i \beta_{k}$, $k=1, \ldots M$, leads again to an amplitude of the form (2.12), where now the prefactor takes the general form

$$
F(E)=\sum_{k=1}^{M} \operatorname{sign}(E) e^{-\beta_{k}|E|+i \alpha_{k} E}
$$


The usual array is a special case of this formula: With $=\infty, \alpha_{k}=0, \beta_{k}=a\left(k-\frac{1}{2}\right)$ we immediately recover (2.13).

All the conclusions of section 3 are valid in this more general case. The basic step is the extraction of the discontinuity in $E$, which localizes the moduli space integration to $\rho \rightarrow 0$. A general configuration of imaginary D-branes leads to a sphere amplitude with an extra closed string insertion $\mathcal{W}$, only the details of this insertion change. All we have to do is to replace $1 / \sinh \left(\frac{|E| a}{2}\right)$ in (3.14) with the general $F(E)$ given in (6.5) $)$. The state $|\mathcal{W}\rangle$ is normalizable and has finite energy as long as all D-branes are at distances $\beta_{k}>\pi$ from the real axis, generalizing the condition $a>2 \pi$ that we had for the array.

For a generic configuration of a finite number of imaginary D-branes, the space-time dependence of $\mathcal{W}$ is, however, quite different than the case of the infinite array. This difference is sharpest for the massless fields outside the lightcone, $r \gg\left|X^{0}\right|$. For the array, they have the same dependence $\sim 1 / r^{23-p}$ as for a static $\mathrm{D} p$-brane, whereas for a finite configuration they decay one power faster $\sim 1 / r^{24-p}$. This nicely dovetails with the fact that the infinite array with $a=2 \pi$ admits the exactly marginal deformation that creates an actual $\mathrm{D} p$ - brane in real time. The incoming and outgoing radiation that makes up $\mathcal{W}_{\text {array }}$ are precisely tuned to admit the deformation that reconstructs the brane, whereas for a generic finite configuration, brane creation would require an abrupt change of the field asymptotics.

It is worth pointing out that our prescription to compute the closed string fields associated with imaginary D-branes is equivalent to the second-quantized point of view taken in section 3 of [12]. They propose to obtain the wavefunction for the closed string fields at $X^{0}=0$ by cutting open the Euclidean path integral in the presence of D-brane sources located at Euclidean time $\widetilde{X}^{0}<0$. To evaluate the expectation value of the closed string fields in such a wave function, one needs to construct the full configuration of sources symmetric under $\widetilde{X}^{0} \rightarrow-\widetilde{X}^{0}$ (obtained by simply reflecting the sources located at $\widetilde{X}^{0}<0$ to $\widetilde{X}^{0}>0$ ), and read off the solution at $\widetilde{X}^{0}=0$. This is the second-quantized version of what we do here.

An interesting open question is whether as we vary the configuration of imaginary D-branes, the state $|W\rangle$ spans the full closed string cohomology. It is clear that to have any chance of success we must introduce more general boundary states than the ones considered in this paper (for example, D-branes with magnetic and electric fields on their worldvolume), and allow for an infinite number of imaginary branes. If the answer to 
this question is in the affirmative then the open/closed string duality takes a new form since at the fundamental level closed strings and D-branes are unified. Each closed string puncture in a string theory amplitude could be effectively represented by a hole in the worldsheet with appropriate boundary conditions.

\subsection{Superstring}

This general set-up can be generalized to the superstring in a straightforward way. There is now more variety of D-brane sources, since we can consider both stable BPS D $p$-branes and unstable non-BPS D $p$-branes. For non-BPS branes the discussion is completely analogous to the bosonic case. One can distribute non-BPS brane pairs freely in the complex $X^{0}$ plane, subject to exactly the same reality condition as we discussed in section 5 .

BPS D $p$ branes introduce on the other hand an important novelty: They are sources of Ramond-Ramond fields. The reality condition for the RR fields forces us to consider pairs composed of a BPS brane at $X^{0}=\alpha+i \beta$ and of its anti-brane partner at $X^{0}=\alpha-i \beta$. It can be checked that the RR fields produced by a generic configuration of such pairs are non-vanishing only inside the lightcone, $r \ll\left|X^{0}\right|$ (see also [11]); this is consistent with the fact that the configuration has zero total $R R$ charge, so we do not see long range $R R$ fields.

Like in the bosonic string, infinite 'critical' arrays of branes at $X^{0}=i a_{\text {crit }}\left(n+\frac{1}{2}\right)$ correspond to special limits of BCFTs related to real time processes of brane creation

and annihilation. Here $a_{c r i t}=\sqrt{2} \pi$ (this is the familiar $\sqrt{2}$ in translating between the bosonic string and the superstring). There are two interesting classes of examples. One can consider a critical array of non-BPS $\mathrm{D}(p-1)$ branes in imaginary time, which is dual to the closed string background related to the decay of a $\mathrm{D} p-\overline{\mathrm{D}} p$ pair; or a critical array of alternating $\operatorname{BPS} \mathrm{D}(p-1) / \overline{\mathrm{D}}(p-1)$ in imaginary time, related to the decay of non-BPS $\mathrm{D} p$. (Needless to say, given $p$, any of these examples makes sense in either Type IIA or IIB, but not in both). The subject is potentially quite rich.

\section{Open string field theory}

We have found an intriguing relation between D-branes in imaginary time and purely closed backgrounds. Since D-branes admit an open string description, this suggests that one may be able to obtain a dual description of closed string theories in terms of open 
strings. We have already seen in section 5 that the exactly marginal open string deformations have a natural re-interpretation as deformations of the closed string background. However since there are no propagating on-shell open string degrees of freedom on imaginary D-branes, it is clear that if such a complete open/closed duality exists, it must involve the off-shell open strings. In this section we offer some very brief and incomplete speculations in this direction.

We would like to propose that the open string field theory (OSFT) on a configuration of imaginary D-branes is dual to the corresponding closed string theory. To make sense of this speculation we must define what is the OSFT for imaginary branes. Applying our usual strategy, we start with OSFT on standard D-branes, and double Wick rotate. While from a first quantized point of view it may be subtle to define the Wick-rotated open string theory, in the second quantized approach we have the luxury of a space-time action, which seems straightforward to analytically continue.

Let us sketch how this may come about in the example of the array of $\mathrm{D}(-1)$ branes. We start with an array of $\mathrm{D}(-1)$ branes at $\widetilde{X}^{0}=\left(n+\frac{1}{2}\right) a$. The open string field $\Psi_{j k}$ has Chan-Paton labels $j, k \in \mathbf{Z}$ running over the positions of the $\mathrm{D}(-1)$ branes, and the cubic OSFT action [35] takes the form

$$
S[\Psi]=-\frac{1}{g_{0}^{2}}\left(\frac{1}{2} \sum_{j k}\left\langle\Psi_{j k}, Q_{B} \Psi_{k j}\right\rangle+\frac{1}{3} \sum_{j k l}\left\langle\Psi_{j k}, \Psi_{k l}, \Psi_{l j}\right\rangle+\sum_{j}\left\langle\Psi_{j j}, \mathcal{C}\right\rangle\right) .
$$

Here we have also included the gauge-invariant open/closed vertex [36, 37, 38, 39, 23 ] $\langle\Psi, \mathcal{C}\rangle$ that couples external on-shell closed strings $\mathcal{C}$ to the open string field. Notice that the string fields $\Psi_{i j}$ do not depend at all on the zero modes of the space-time coordinates. Double Wick rotation is then immediate. There is little to do in all the purely open terms of the action. ${ }^{15}$ Only the open/closed vertex is affected. We conjecture that the resulting action describes the non-trivial closed string state $|W\rangle$.

In principle it should be possible to recover the results of section 3 from the point of view of the second quantized Feynman rules for the action (7.1). In OSFT, amplitudes of external closed string on the disk are obtained by computing expectation values of the gauge-invariant operators discussed in [36, 37, 38, 39, 23, or in other terms by the use of the open/closed vertex. We expect that for (17.1) such amplitudes collapse to the

\footnotetext{
${ }^{15}$ Note however that one has to be careful here with the reality conditions discussed in the previous sections [40.
} 
region of moduli space where the open string propagators have zero length, reducing to sphere amplitudes with an extra insertion of $|W\rangle$. The mechanism for such a collapse must be of a somewhat different nature than in [23] or in [41], since here we are using the conventional BRST operator but a highly unconventional state-space.

Finally, the OSFT (7.1) may provide us with new clues about the string field theory around the tachyon vacuum. As $a \rightarrow \infty$, the energy of $|W\rangle$ goes to zero, and we approach the tachyon vacuum. Interestingly, in the same limit $|W\rangle$ does not vanish completely, but it becomes purely a zero-momentum dilaton. Thus we recover precisely the scenario of 23. Since for any $a$ such an open string field theory should make sense this may provide us with a consistent regularization of vacuum string field theory, which should be related to the one considered in [23] by some non-trivial field redefinition.

\section{$8 \mathrm{a}=2 \pi$ and reconstruction of the brane}

As discussed in section 4, for $a=2 \pi$ the normalization and the energy associated with the closed string state $|\mathcal{W}\rangle$ diverge [12]. This singularity signals the appearance of new open string degrees of freedom. Open strings stretched between neighboring branes in imaginary time have conformal dimension $L_{0}=(a / 2 \pi)^{2}$, which equals one for $a=2 \pi$. The infinite periodic array has the special property that a specific linear combination of these marginal operators (the one which is invariant under $X_{0} \rightarrow X_{0}+2 \pi i$ and is even under $\left.X_{0} \rightarrow-X_{0}\right)$ is in fact exactly marginal ${ }^{16}$. A new branch of moduli space opens up for $a=2 \pi$. Indeed, the theory for $a=2 \pi$ is equivalent to the $\lambda=\frac{1}{2}$ critical point of the BCFT (1.1). Turning on the exactly marginal open string deformation (' $\left.\cosh \left(X^{0}\right)^{\prime}\right)$ we can reduce the value of $\lambda$, from $\lambda=\frac{1}{2}$ (the purely closed string background $|W\rangle$ ) to $\lambda=0$ (the usual brane with Neumann boundary condition in time). This is the sense in which the array for $a=2 \pi$ is very special: it admits an exactly marginal deformation that cannot be interpreted purely as a deformation of the closed background.

\subsection{Smearing and brane creation}

One can obtain many insights into the physics for $\lambda<1 / 2$ by a simple extension of the methods in section 2. The basic idea is that in the Euclidean BCFT with $X=-i X^{0}$,

\footnotetext{
${ }^{16}$ In principle also the odd operator, ' $\sinh \left(X^{0}\right)$ ', is exactly marginal. However in the bosonic string this deformation would bring us to the 'wrong' side of the tachyon potential for $X^{0}<0$.
} 
taking $\lambda<\frac{1}{2}$ amounts to regulating the delta-function sources located at $X=2 \pi(n+1 / 2)$ with smooth lumps. The precise way to make this regulation can be gleaned from the boundary state 25] for general $\lambda$. Focusing on the oscillator free part of the boundary state,

$$
\left|\mathcal{B}_{0}\right\rangle \sim\left[1+2 \sum_{n=1}^{\infty}(-1)^{n} e^{-n \tau} \cos (n X(0))\right]|0\rangle, \quad \tau \equiv-\log (\sin (\pi \lambda)) .
$$

By Poisson resummation, one finds

$$
\left|\mathcal{B}_{0}\right\rangle \sim \sum_{-\infty}^{\infty} \tilde{j}_{\tau}\left(X+2 \pi\left(n+\frac{1}{2}\right)\right)|0\rangle, \quad \tilde{j}_{\tau}(X)=\frac{\tau}{\pi\left(X^{2}+\tau^{2}\right)}
$$

The interpretation of this formula is clear: for $\lambda<\frac{1}{2}$, the boundary state corresponds to an infinite array of smeared sources $\tilde{j}_{\tau}$; indeed $\tilde{j}_{\tau}$ is a well-known representation for $\delta(X)$ in the limit $\tau \rightarrow 0$.

If we sum the source $\tilde{j}_{\tau}(X)$ over the infinite array and then Wick rotate $X \rightarrow-i X^{0}$ we find

$$
J_{\tau}\left(X^{0}\right)=\frac{\tanh \left(\left(X^{0}+\tau\right) / 2\right)-\tanh \left(\left(X^{0}-\tau\right) / 2\right)}{4 \pi} .
$$

Already at this stage we see the crucial difference compared to $\lambda=\frac{1}{2}(\tau=0)$. Now there is a non-zero source localized at real time for $\left|X^{0}\right|<\tau$, which is to say that an unstable D-brane appears at $X^{0}=-\tau$ and disappears at $X^{0}=\tau$. For later use let us record the Fourier transform of this function,

$$
\rho_{\tau}(E)=\frac{\sin (\tau E)}{\sinh (\pi E)} .
$$

The next step is to repeat the exercise for the fields generated by this smeared array. The Fourier transform of the Euclidean source $\tilde{j}_{\tau}(X)$ is $e^{-\tau|P|}$. Therefore, in the notations of section 2.2, eq.(2.4) should be replaced by

$$
\tilde{A}(P, \ldots)=\frac{e^{-\tau|P|}}{P^{2}+c^{2}} .
$$

As in section 2.3, we sum over the array and use the residue theorem to write the total field as a contour integral,

$$
\widetilde{G}_{\tau}(X)=\frac{1}{2 i} \oint_{\mathcal{C}} d P e^{i P X} \frac{e^{-\tau|P|}}{\left(P^{2}+c^{2}\right) \sin (\pi P)} .
$$


If we now move the contour over the imaginary $P$ axis as in Fig.2, and Wick rotate, we find

$$
S_{\tau}(E)=\frac{1}{2 \sinh (\pi E)}\left(\frac{e^{i \tau E}}{(E+i \epsilon)^{2}-c^{2}}-\frac{e^{-i \tau E}}{(E-i \epsilon)^{2}-c^{2}}\right),
$$

which we recognize as

$$
S_{\tau}(E)=\frac{1}{2 \sinh (\pi E)}\left(G_{r e t}(E) e^{i \tau E}-G_{a d v}(E) e^{-i \tau E}\right) .
$$

This way of writing the answer makes the space-time interpretation manifest (see also [12]). For $X^{0}<-(\tau+r)$, we have purely incoming radiation. For $X^{0}>\tau+r$, we have purely outgoing radiation. Outside of these two cones (for $\left(X^{0}\right)^{2}-r^{2}<\tau^{2}$ ), the fields are the same as the ones produces by a static source. The thickness of the transition regions is of the order of the string length. Notice that the outgoing radiation is produced by the rapid change of the source for $X^{0} \sim \tau$, and similarly the incoming radiation is correlated with the change at $X^{0} \sim-\tau$. This process can be described as some finely tuned incoming closed strings that create an unstable D-brane at $X^{0}=-\tau$, which then decays at $X^{0}=\tau$ into outgoing closed strings.

We can also write

$$
S_{\tau}(E, \ldots)=G_{F}(E) \rho_{\tau}(E)+\cos (\tau E) \frac{\pi}{2 c \sinh \left(\frac{c a}{2}\right)}(\delta(E-c)+\delta(E+c)),
$$

where $G_{F}(E)$ is the Feynman propagator and $\rho(E)$ the Fourier transform of the source in real time $J_{\tau}\left(X^{0}\right)$, see (8.4). The two terms in (8.9) have very different interpretations. While the first term contains a propagator, and is non-zero for any finite $E$, the second term has support only for $E= \pm c$. The second term in (8.9) is proportional to (2.9); in section 3 it was seen to correspond to the extra insertion $|W\rangle$ in a sphere amplitude. The first term is instead a real D-brane source, associated to an earnest disk amplitude.

In the limit $\lambda \rightarrow \frac{1}{2}(\tau \rightarrow 0)$ only the second term in (8.9) contributes, since the source $\rho_{\tau}(E) \rightarrow 0$. It is a bit less obvious to see why only the first term contributes as $\lambda \rightarrow 0$. In this limit, $\tau \rightarrow \infty$ and since the oscillations of both terms are controlled by the dimensionless parameter $\tau E$, to have a non-zero contribution $E$ must go to zero, hence for any fixed finite $c$ the second term vanishes. The fact the $E \rightarrow 0$ is consistent with the fact that the for $\lambda=0$ the unstable D-brane exists for an infinite amount of time; $X^{0}$ becomes an ordinary Neumann direction and the total energy (with all insertions as incoming) must vanish. 
We see that as we vary $\lambda$, not only does the D-brane source vary, but the closed string background (captured by the second term in (8.9)) also changes in a very nontrivial way. This phenomenon requires some comments. Although the BCFT (1.1) may be naively thought of as a deformation of the open string background only, infact we need to simultaneously change the closed string background to cancel tadpoles. Indeed, while the operator $\cosh \left(X^{0}\right)$ is exactly marginal in the open string sense, one point functions of generic on-shell closed strings have a non-trivial dependence on $\lambda$. Of course, tadpoles for closed string operators are also generated in the familiar case of time-independent boundary deformations. However in the time-independent case only one-point functions of zero-energy on-shell bulk operators can change, whereas for a time-dependent perturbation like (1.1) there are tadpoles with a non-trivial space-time dependence. As we turn on the cosh deformation, the closed string background needs to be corrected introducing closed string matter. Happily, as (8.9) shows, this seems to be automatically taken care of by the analytic continuation procedure.

The discussion in this section has been at the level of the discussion of section 2 . It would be very interesting to repeat the analysis of section 3 and compute scattering amplitudes of closed strings for general $\lambda$. It would also be of interest to find the behavior of the open string spectrum for $0<\lambda<\frac{1}{2}$.

\section{From Choptuik to Gregory-Laflamme}

As was explained in section 4 the massless sector of $|W\rangle$ is a spherically symmetric dilaton wave in $26-p$ dimensions. In the limit that the Newton constant $G_{N}$ goes to zero the linearized solution is an exact solution to the gravity-dilaton equations of motion. As the coupling constant is turned on the non-linearity of the equation of motion becomes more and more important. With spherical symmetry much is known about the non-linear aspects of the system. In particular, Choptuik showed [27], via numerical analysis, that a universal behavior occurs at the critical point where black hole formation first occurs. A crude summary of his results is the following. Consider a spherically symmetric wave of a massless scalar field

$$
\eta \phi\left(r, X^{0}\right)
$$

The strength of the non-linear effects of the gravitational back-reaction of the wave grows with the overall coefficient $\eta$. When $\eta \rightarrow 0$ the linearized approximation is exact while for 
$\eta \rightarrow \infty$ a large black hole will be formed, with an exponentially small amount of energy escaping the black hole formation as an outgoing radiation. ${ }^{17}$ Therefore, for a given field profile $\phi\left(r, X^{0}\right)$ there is a special value $\eta^{*}$ where the black hole formation first takes place. While $\eta^{*}$ certainly depends on the details of $\phi$, the time evolution of the system for $\eta \rightarrow \eta^{*}$ admits scaling behavior that is fixed by a certain constant, $\Delta$. This constant is universal in the sense that it does not depend on $\phi$, but it can depend for example on the number of spatial directions. Another critical exponent $\delta$ is related to the scaling of the mass of the black hole near the transition. These fascinating results have been explored quite extensively in the last decade.

In this section we take advantage of the fact that a spherically symmetric dilaton wave can be viewed as a configuration of D-branes located in imaginary time to relate Choptuik's findings to a phase transition somewhat reminiscent of the Gregory-Laflamme instability 28. For simplicity we will mostly phrase the discussion in the context of the bosonic string, ignoring as usual the closed string tachyon. The extension to the superstring of the scaling arguments given below is straightforward.

The first step is to understand how to get from our $|W\rangle$ a background containing only a classical dilaton wave. It is clear that to suppress massive closed string modes we need to take $\frac{a}{l_{s}} \rightarrow \infty$. (Here we have restored the string length $l_{s}$, that was set to one in the rest of the paper). However the total energy of the wave will then go to zero as $1 / a^{25-p}$ in this limit (see (4.9)), and a black hole will not be formed this way. A simple way to obtain a configuration with enough energy without exciting the massive modes is to increase the number of branes at each point $X^{0}=i a\left(n+\frac{1}{2}\right)$, as in the discussion at the end of section 4. With $N$ D-brane at each point the particles density, $\bar{n} / V$, and the energy density $\epsilon=\bar{E} / V$ ( $V$ is the volume along the wave) of the wave for large $a$ are (from (4.9))

$$
\frac{\bar{n}}{V} \sim \frac{N^{2} l_{s}^{24-2 p}}{a^{24-p}}, \quad \epsilon \sim \frac{N^{2} l_{s}^{24-2 p}}{a^{25-p}} .
$$

Let us now state the exact conditions that must be satisfied in the limit we want to take:

1. The string coupling $g_{s}$ should go to zero to suppress quantum effects, and the total number of particles $\bar{n}$ should go to infinity so that $|W\rangle$ is well described by a classical wave.

\footnotetext{
${ }^{17}$ The system is classical so this radiation is classical and should not be confused with Hawking radiation.
} 
2. $\frac{l_{s}}{a} \rightarrow 0$ to ensure that the massive closed strings decouple.

3. The gravitational radius associated with the total energy of the wave,

$$
r_{G}=\left(G_{N} \epsilon\right)^{\frac{1}{23-p}},
$$

(here $G_{N}$ is the Newton constant in 26 dimensions) should be comparable to the wavelength $a$. If we define the dimensionless parameter

$$
\zeta=\frac{r_{G}}{a}=\frac{\left(g_{s}^{2} N^{2} l_{s}^{48-2 p} / a^{25-p}\right)^{\frac{1}{23-p}}}{a}
$$

then in our set-up $\zeta$ plays the role of $\eta$ in (9.1) That is, for small $\zeta$ the linearized approximation is valid and a black hole will not be formed while for large $\zeta$ nonlinear effects are important and lead to black hole formation. So the critical value $\zeta^{*}$ is a number of order one.

It is easy to verify that these conditions are satisfied in the following limit

$$
\text { a fixed, } \quad l_{s} \rightarrow 0, \quad g_{s} \propto l_{s}^{\beta}, \quad N \propto 1 / l_{s}^{24+\beta-p}, \quad \beta>0 .
$$

-So far what we have done is to take a certain 'decoupling' limit that leaves us with the classical picture of black-hole formation. The obvious question to ask is what does this mean from the D-brane point of view. In particular, does anything special happen at $\zeta=\zeta^{*}$ in the array of $\mathrm{D}(p-1)$ branes located at $\widetilde{X}^{0}=\left(n+\frac{1}{2}\right)$ a, i.e. in the spatial array before the double Wick rotation?

The gravitational radius associated with $\mathrm{N}$ D-branes located at each site is

$$
l_{G} \sim\left(g_{s} N\right)^{\frac{1}{24-p}} l_{s}
$$

When $l_{G}$ is much smaller than the distance between the D-branes, $a$, the gravitational interactions between them is small and they can be considered as separated points. However, when $l_{G}$ is larger than $a$ the gravitational interaction between them is so strong that effectively they form a black hole along $\widetilde{X}^{0}$. It is easy to see from (9.69.4) that this transition occurs at the same point as the wave to black hole transition. Since the two processes are related by Wick rotation it is very tempting to suspect that there is a precise numerical relation between the exponents of [27] and the exponents, yet to be found, in the phase transition just described. 
It should be stressed that although the transition considered here is somewhat reminiscent of the Gregory-Laflamme instability, there are also obvious differences. In the Gregory-Laflamme case one starts with a black $p$-brane and finds, at the level of linearized equations of motion, an instable mode; condensation of this tachyon is then conjectured to lead to an array of black ( $p$-1)-branes. (This expectation however may even be false, see e.g. [42, 43, 44 for recent discussions). In our case we start from the array and we turn on the coupling constant to eventually form a (possibly non-uniform) black $p$ brane, so we are going in the opposite direction. More crucially, we do not start with an array of black p-branes. The D-branes are extremal to begin with, in the sense that they have no finite area horizon. In the process of turning on the coupling constant the radius of their zero area horizon grows until they meet. At that point a combined finite area horizon will be formed.

Let us illustrate this in the context of the superstring. In this case there are no closed string tachyons so the whole discussion makes more sense. We can consider either an array of BPS D $(p-1)$-branes, which must then be alternating as $\mathrm{D} / \overline{\mathrm{D}}$, or an array of non-BPS $\mathrm{D}(p-1)$ branes. Consider for example an array of D3/D̄3 in Type IIB. For small $\zeta$ the D3 branes interact weakly and so they can be treated as separated stable objects. As we turn on $\zeta$ their gravitational radius start to touch. At that stage the fact that we have alternating branes anti-branes is crucial since the charges can annihilated to form a black 4-brane. This black 4-brane does not carry any 6-form charge (we are in type IIB).

It would be extremely interesting to understand Choptuik's critical behavior from the point of view of the dual open string field theory discussed very briefly in section 7 . Very schematically, the way this could work is as follows. From the open string field theory point of view the classical non-linear gravity dynamics is obtained in the loop expansion. We expect that the lightest open string mode stretched between the branes, which at the classical level has large positive $m^{2}$, becomes tachyonic for $\zeta>\zeta^{*}$ due to quantum open string effects. It may be possible to see this effect in a one-loop computation, in the spirit of [45]. If this scenario is correct, the universal physics at the transition point would be completely captured by a massless field, and we would have an explanation of gravitational critical behavior in terms of a second order phase transition in the dual OSFT. 


\section{Acknowledgements}

We thank C. Callan, A. Hashimoto, I. Klebanov, H. Liu, J. Maldacena, J. McGreevy, H. Ooguri, A. Sen, C. Vafa and H. Verlinde for discussions. This material is based upon work supported by the National Science Foundation under Grant No. PHY 9802484. Any opinions, findings, and conclusions or recommendations expressed in this material are those of the author and do not necessarily reflect the views of the National Science Foundation.

\section{References}

[1] M. Gutperle and A. Strominger, "Spacelike branes," JHEP 0204, 018 (2002) arXiv:hep-th/0202210.

[2] A. Sen, "Rolling tachyon," JHEP 0204, 048 (2002) arXiv:hep-th/0203211.

[3] A. Sen, "Tachyon matter," JHEP 0207, 065 (2002) arXiv:hep-th/0203265.

[4] A. Sen, "Field theory of tachyon matter," Mod. Phys. Lett. A 17, 1797 (2002) arXiv:hep-th/0204143.

[5] A. Sen, "Time evolution in open string theory," JHEP 0210, 003 (2002) arXiv:hep-th/0207105.

[6] P. Mukhopadhyay and A. Sen, "Decay of unstable D-branes with electric field," JHEP 0211, 047 (2002) arXiv:hep-th/0208142.

[7] A. Strominger, "Open string creation by S-branes," arXiv:hep-th/0209090.

[8] A. Sen, "Time and tachyon," arXiv:hep-th/0209122.

[9] F. Larsen, A. Naqvi and S. Terashima, "Rolling tachyons and decaying branes," JHEP 0302, 039 (2003) arXiv:hep-th/0212248.

[10] M. Gutperle and A. Strominger, "Timelike boundary Liouville theory," arXiv:hep-th/0301038

[11] A. Maloney, A. Strominger and X. Yin, "S-brane thermodynamics," arXiv:hep-th/0302146. 
[12] N. Lambert, H. Liu and J. Maldacena, "Closed strings from decaying D-branes," arXiv:hep-th/0303139

[13] B. Chen, M. Li and F. L. Lin, "Gravitational radiation of rolling tachyon," JHEP 0211, 050 (2002) arXiv:hep-th/0209222.

[14] S. J. Rey and S. Sugimoto, "Rolling tachyon with electric and magnetic fields: Tduality approach," arXiv:hep-th/0301049.

[15] N. Moeller and B. Zwiebach, "Dynamics with infinitely many time derivatives and rolling tachyons," JHEP 0210, 034 (2002) arXiv:hep-th/0207107.

[16] S. Sugimoto and S. Terashima, "Tachyon matter in boundary string field theory," JHEP 0207, 025 (2002) arXiv:hep-th/0205085.

[17] J. A. Minahan, "Rolling the tachyon in super BSFT," JHEP 0207, 030 (2002) arXiv:hep-th/0205098.

[18] T. Okuda and S. Sugimoto, "Coupling of rolling tachyon to closed strings," Nucl. Phys. B 647, 101 (2002) arXiv:hep-th/0208196.

[19] J. Kluson, "Exact solutions in open bosonic string field theory and marginal deformation in CFT," arXiv:hep-th/0209255.

[20] I. Y. Aref'eva, L. V. Joukovskaya and A. S. Koshelev, "Time evolution in superstring field theory on non-BPS brane. I: Rolling tachyon and energy-momentum conservation," arXiv:hep-th/0301137.

[21] A. Ishida and S. Uehara, "Rolling down to D-brane and tachyon matter," JHEP 0302, 050 (2003) arXiv:hep-th/0301179.

[22] S. L. Shatashvili, "On field theory of open strings, tachyon condensation and closed strings," arXiv:hep-th/0105076.

[23] D. Gaiotto, L. Rastelli, A. Sen and B. Zwiebach, "Ghost structure and closed strings in vacuum string field theory," arXiv:hep-th/0111129.

[24] L. Rastelli, A. Sen and B. Zwiebach, "String field theory around the tachyon vacuum," Adv. Theor. Math. Phys. 5, 353 (2002) arXiv:hep-th/0012251. 
[25] C. G. Callan, I. R. Klebanov, A. W. Ludwig and J. M. Maldacena, "Exact solution of a boundary conformal field theory," Nucl. Phys. B 422, 417 (1994) arXiv:hep-th/9402113.

[26] J. Polchinski and L. Thorlacius, "Free Fermion Representation Of A Boundary Conformal Field Theory," Phys. Rev. D 50, 622 (1994) arXiv:hep-th/9404008.

[27] M. W. Choptuik, "Universality And Scaling In Gravitational Collapse Of A Massless Scalar Field," Phys. Rev. Lett. 70, 9 (1993).

[28] R. Gregory and R. Laflamme, "Black Strings And P-Branes Are Unstable," Phys. Rev. Lett. 70, 2837 (1993) arXiv:hep-th/9301052.

[29] I. R. Klebanov and L. Thorlacius, "The Size of p-Branes," Phys. Lett. B 371, 51 (1996) arXiv:hep-th/9510200.

[30] A. Hashimoto and I. R. Klebanov, "Decay of Excited D-branes," Phys. Lett. B 381, 437 (1996) arXiv:hep-th/9604065.

[31] J. Polchinski, "String Theory Vol. 1" Cambridge University Press (1995).

[32] B. Zwiebach, "Closed string field theory: Quantum action and the B-V master equation," Nucl. Phys. B 390, 33 (1993) arXiv:hep-th/9206084.

[33] P. Di Vecchia, M. Frau, I. Pesando, S. Sciuto, A. Lerda and R. Russo, "Classical pbranes from boundary state," Nucl. Phys. B 507, 259 (1997) arXiv:hep-th/9707068.

[34] M. Green, J. Schwarz and E.Witten, "Superstring Theory Vol. 1" Cambridge University Press (1987).

[35] E. Witten, "Noncommutative Geometry And String Field Theory," Nucl. Phys. B 268, 253 (1986).

[36] J. A. Shapiro and C. B. Thorn, "Brst Invariant Transitions Between Closed And Open Strings," Phys. Rev. D 36, 432 (1987).

[37] J. A. Shapiro and C. B. Thorn, "Closed String - Open String Transitions And Witten's String Field Theory," Phys. Lett. B 194, 43 (1987). 
[38] B. Zwiebach, "Interpolating string field theories," Mod. Phys. Lett. A 7, 1079 (1992) arXiv:hep-th/9202015.

[39] A. Hashimoto and N. Itzhaki, "Observables of string field theory," JHEP 0201, 028 (2002) arXiv:hep-th/0111092.

[40] Work in progress.

[41] N. Drukker, "Closed string amplitudes from gauge fixed string field theory," arXiv:hep-th/0207266.

[42] G. T. Horowitz and K. Maeda, "Fate of the black string instability," Phys. Rev. Lett. 87, 131301 (2001) arXiv:hep-th/0105111.

[43] S. S. Gubser, "On non-uniform black branes," Class. Quant. Grav. 19, 4825 (2002) arXiv:hep-th/0110193.

[44] B. Kol, "Topology change in general relativity and the black-hole black-string transition," arXiv:hep-th/0206220.

[45] S. R. Coleman and E. Weinberg, "Radiative Corrections As The Origin Of Spontaneous Symmetry Breaking," Phys. Rev. D 7, 1888 (1973). 